\title{
An Iteration Method for the Solution of the Eigenvalue Problem of Linear Differential and Integral Operators ${ }^{1}$
}

\section{By Cornelius Lanczos}

\begin{abstract}
The present investigation designs a systematic method for finding the latent roots and the principal axes of a matrix, without reducing the order of the matrix. It is characterized by a wide field of applicability and great accuracy, since the accumulation of rounding errors is avoided, through the process of "minimized iterations". Moreover, the method leads to a well convergent successive approximation procedure by which the solution of integral equations of the Fredholm type and the solution of the eigenvalue problem of linear differential and integral operators may be accomplished.
\end{abstract}

\section{Introduction}

The eigenvalue problem of linear operators is of central importance for all vibration problems of physics and engineering. The vibrations of elastic structures, the flutter problems of aerodynamics, the stability problem of electric networks, the atomic and molecular vibrations of particle physics, are all diverse aspects of the same fundamental problem, viz., the principal axis problem of quadratic forms.

In view of the central importance of the eigenvalue problem for so many ficlds of pure and applied mathematics, much thought has bcen devoted to the designing of efficient methods by which the cigenvalues of a given linear operator may be found. That linear operator may be of the algebraic or of the continuous type; that is, a matrix, a differential operator, or a Fredholm kernel function. Iteration methods play a prominent part in these designs, and the literature on the iteration of matrices is very extensive. ${ }^{2}$ In the English literature of recent years the works of H. Hotelling [1] ${ }^{3}$ and A. C. Aitken [2] deserve attention. H. Wayland [3] surveys the field in its historical development, up to recent years. W. U. Kincaid [4] obtained additional results by improving the convergence of some of the classical procedures.

I The preparation of this paper was sponsored in part by the Office of Naval Research.

2 The basic principjes of the various iteration methods are exhsustively treated in the well-known book on Elementary matrices by R, A, Frazer, W. J. Dunean, and A. R. Collar (Cambridge University Press, 1938); (MacMillan, New York, N. Y, 1947).

a Floures In hrackets indieate the litersture relerenees at the end of this paper.

$904428-50-1$
The present investigation, although starting out along classical lines, proceeds nevertheless in a different direction. The advantages of the method here developed ${ }^{*}$ can be summarized as follows:

1. The iterations are used in the most economical fashion, obtaining an arbitrary number of eigenvalues and eigensolutions by one single set of iterations, without reducing the order of the matrix.

2. The rapid aceumulation of fatal rounding errors, common to all iteration processes if applied to matrices of high dispersion (large "spread" of the eigenvalues), is effectively counteracted by the method of "minimized iterations".

3. The method is directly translatable into analytical terms, by replacing summation by integration. We then get a rapidly convergent analytical iteration process by which the eigenvalues and eigensolutions of linear differential and integral equations may be obtained.

\section{The Two Classical Solutions of Fredholm's Problem}

Since Fredholm's fundamental essay on integral equations [5], we can replace the solution of linear differential and integral equations by the solution

\footnotetext{
1 The literature svailable to the author showed no evidence that the methods and results of the presunt investigation have been found before. Fowever, A. M. Ostrowski of the University of Basle and the Institute for Nunerical Analysis informed the author that bls method paraliols the earlicr work of some Russian sclentists; the references given by Ostrowski are: A, Krylov, Izv. Aksd. Sisuk SSSR 7, 491 to 539 (1931); N. Luzin, Izv. Akad. Nauk SSSR 7, 903 to 958 (1931). On the basis of the reviews of these pspers in the Zentralbistt, the author believes that the two methods coincide only in the point of departure. The author bus not, bowever, read these Russian papers.
} 
of a set of simultaneous ordinary linear equations of infinite order. The problem of Fredholm, if formulated in the language of matrices, can be stated as follows: Find a solution of the equation

$$
y-\lambda A y=b,
$$

where $b$ is a given vector, $\lambda$ a given scalar parameter, and $A$ a given matrix (whose order eventually approaches infinity); whereas $y$ is the unknown vector. The problem includes the inversion of a matrix $(\lambda=\infty)$ and the problem of the characteristic solutions, also called "eigensolutions", $(b=0)$ as special cases.

Two fundamentally different classical solutions of this problem are known. The first solution is known as the "Liouville-Neumann expansion" [6]. We consider $A$ as an algebraic operator and obtain formally the following infinite geometric series:

$$
y=\frac{1}{1-\lambda A} b=\left(1+\lambda A+\lambda^{2} A^{2}+\ldots\right) b .
$$

This series converges for sufficiently small values of $|\lambda|$ but diverges beyond a certain $|\lambda|=\left|\lambda_{1}\right|$. The solution is obtained by a series of successive "iterations"; ${ }^{s}$ we construct in succession the following set of vectors:

$$
\left.\begin{array}{c}
b_{0}=b \\
b_{1}=A b_{0} \\
b_{2}=A b_{1} \\
\vdots \\
\cdot \\
b_{n+1}=A b_{n}
\end{array}\right\}
$$

and then form the sum:

$$
y=b_{0}+\lambda b_{1}+\lambda^{2} b_{2}+\ldots .
$$

The merit of this solution is that it requires nothing but a sequence of iterations. The drawback of the solution is that its convergence is limited to sufficiently small values of $\lambda$.

The second classical solution is known as the Schmidt series [7]. We assume that the matrix $A$ is "nondefective" (i. e. that all its elementary divisors are linear). We furthermore assume that

"Throughont this paper the term "iteration" refers to the spplication of the given matrix $A$ to a given vector $b$, by forming the product $A b$. we possess all the eigenvalues ${ }^{b} \mu_{i}$ and eigenvectors $u_{i}$ of the matrix $A$, defined by the equations

$$
A u_{i}=\mu_{i} u_{i} \quad(i=1,2, \ldots, n) .
$$

If $A$ is nonsymmetric, we need also the "adjoint" eigenvectors $u_{\hat{\imath}}{ }^{*}$, defined with the help of the transposed matrix $A^{*}$ :

$$
A^{*} u_{i}{ }^{*}=\mu_{i} u_{i}^{*} \quad(i=1,2, \ldots, n) .
$$

We now form the scalars

$$
\gamma_{i}=\frac{b \cdot u_{i}^{*}}{u_{i} \cdot u_{i}^{*}}
$$

and obtain $y$ in form of the following expansion:

$$
y=\frac{\gamma_{1} u_{1}}{1-\lambda \mu_{1}}+\frac{\gamma_{2} u_{2}}{1-\lambda \mu_{2}}+\cdots+\frac{\gamma_{n} u_{n}}{1-\lambda \mu_{n}}
$$

This series offers no convergence difficulties, since it is a finite expansion in the case of matrices of finite order and yields a convergent expansion in the case of the infinite matrices associated with the kernels of linear differential and integral operators.

The drawback of this solution is-apart from the exclusion of defective matrices ${ }^{7}$-that it presupposes the complete solution of the eigenvalue problem associated with the matrix $A$.

\section{Solution of the Fredholm Problem by the S-Expansion}

We now develop a new expansion that solves the Fredholm problem in similar terms as the Liouville-Neumann series but avoids the convergence difficulty of that solution.

We first notice that the iterated vectors $b_{0}, b_{1}$, $b_{2}$, ... cannot be linearly independent of each other beyond a certain definite $b_{k}$. All these vectors find their place within the $n$-dimensional space of the matrix $A$, hence not more than $n$ of them can be linearly independent. We thus know in advance that a linear identity of the following form must exist between the successive iterations.

$$
b_{m}+g_{1} b_{m-1}+g_{2} b_{m-2}+\cdots+g_{m} b_{0}=0 .
$$

"We shall use the term "eigenvalue" for the numbers $m$ defined by (5), whereas the reciprocals of the efgonvalues: $\lambda_{i}=1 / \mu_{i}$ shall be called "character, Istie numbers".

7 The characteristic solutions of defective matrices (1. e. matrices whose elementary divisors are not throughout linear) do not include the entire $n$-dimensional space, strice such Inatrices possess less than $n$ independent princips] sxes. 
We cannot tell in advance what $m$ will be, except for the lower and upper bounds:

$$
1 \leqq m \leqq n .
$$

How to establish the relation (9) by a systematic algorithm will be shown in section VI. For the time being we assume that the relation (9) is already established. We now define the polynomial

$$
G(x)=x^{m}+g_{1} x^{m-1}+\cdots+g_{m},
$$

together with the "inverted polynomial" (the coefficients of which follow the opposite sequence):

$$
S_{m}(\lambda)=1+g_{1} \lambda+g_{2} \lambda^{2}+\cdots+g_{m} \lambda^{m} .
$$

Furthermore, we introduce the partial sums of the latter polynomial:

$$
\left.\begin{array}{rl}
S_{0} & =1 \\
S_{1}(\lambda) & =1+g_{1} \lambda \\
S_{2}(\lambda) & =1+g_{1} \lambda+g_{2} \lambda^{2} \\
\cdot & \\
\cdot \\
S_{m-1}(\lambda) & =1+g_{1} \lambda+\cdots+g_{m-1} \lambda^{m-1}
\end{array}\right\}
$$

We now refer to a formula which can be proved by straightforward algebra: ${ }^{8}$

$$
\begin{aligned}
\frac{S_{m}(\lambda)-\lambda^{m} G(x)}{1-\lambda x} & =S_{m-1}(\lambda)+S_{m-2}(\lambda) \cdot \lambda x \\
& +S_{m-3}(\lambda) \cdot \lambda^{2} x^{2}+\cdots+S_{0} \cdot \lambda^{m-1} x^{m-1} .
\end{aligned}
$$

Let us apply this formula operationally, replacing $x$ by the matrix $A$, and operating on the vector $b_{3}$. In view of the definition of the vectors $b_{i}$. the relation (9) gives:

$$
G(A) \cdot b_{0}=0,
$$

and thus we obtain:

$\frac{S_{m}(\lambda)}{1-\lambda A} b_{0}=S_{m-1}(\lambda) b_{0}+S_{m-2}(\lambda) \lambda b_{1}+\cdots+S_{0} \lambda^{m-1} b_{m-1}$,

and hence:

$y=\frac{1}{1-\lambda A} b_{0}=\frac{S_{m-1}(\lambda) b_{0}+S_{m-2}(\lambda) \lambda b_{1}+\cdots+\cdots}{S_{m}(\lambda)} S_{0} \lambda^{m-1} b_{m-1}$.

\footnotetext{
In order to prevent this paper from becoming too lengthy the analytical details of the prasent investigation ace kept to a minimum, and in a fow places the reader is requested to interpalate the missing steps.
}

If we compare this solution with the earlier solution (4), we notice that the expansion (17) may be conceived as a modified form of the Liouville-Neumann series, because it is composed of the same kind of terms, the difference being only that we weight the terms $\lambda^{k} b_{k}$ by the weight factors

$$
w_{k}=\frac{S_{m-k-1}(\lambda)}{S_{m}(\lambda)}
$$

instead of taking them all with the uniform weight factor 1 . This weighting has the beneficial effect that the series terminates after $m$ terms, instead of going on endlessly. The weight factors $w_{i}$ are very near to 1 for small $\lambda$ but become more and more important as $\lambda$ increases. The weighting makes the series convergent for all values of $\lambda$.

The remarkable feature of the expansion (17) is its complete generality. No matter how defective the matrix $A$ may be, and no matter how the vector $b_{0}$ was chosen, the expansion (17) is always valid, provided only that we interpret it properly. In particular we have to bear in mind that there will always be $m$ polynomials $S_{k}(\lambda)$, even though every $S_{k}(\lambda)$ may not be of degree $k$, due to the vanishing or the higher coefficients. For example, it could happen that

so that

$$
G(x)=x^{m},
$$

$$
\begin{aligned}
& S_{m}(\lambda)=1+0 \lambda+0 \lambda^{2}+\ldots+0 \lambda^{m}, \\
& S_{k}(\lambda)=1+0 \lambda+0 \lambda^{2}+\ldots+0 \lambda^{k},
\end{aligned}
$$

and the formula (17) gives:

$$
y=b_{0}+\lambda b_{1}+\ldots+\lambda^{m-1} b_{m-1} .
$$

\section{Solution of the Eigenvalue Problem}

The Liouville-Neumann scries cannot give the solution of the eigenvalue problem, since the expansion becomes divergent as soon as the parameter $\lambda$ reaches the lowest characteristic number $\lambda_{1}$. The Schmidt series cannot give the solution of the eigenvalue problem since it presupposes the knowledge of all the eigenvalues and eigenvectors of the matrix $A$. On the other hand, the expansion (17), which is based purely on iterations and yet remains valid for all $\lambda$, must contain implicitly the solution of the principal axis problem. Indeed, let us write the right side of (1) in the form

$$
b=S_{m}(\lambda) \bar{b}
$$


Then the expansion (17) loses its denominator and becomes:

$y=S_{m-1}(\lambda) \bar{b}_{0}+S_{n-2}(\lambda) \lambda \bar{b}_{1}+\ldots+S_{0} \lambda^{m-1} \bar{b}_{m-1}$

We can now answer the question whether a solution of the homogeneous equation

$$
y-\lambda A y=0 \text {, }
$$

is possible without the identical vanishing of $y$. The expression (23) shows that $b$ can vanish only under two circumstances; either the vector $\bar{b}$, or the scalar $S_{m}(\lambda)$ must vanish. Since the former possibility leads to an identically vanishing $y$, only the latter possibility is of interest. This gives the following condition for the parameter $\lambda$.

$$
S_{m}(\lambda)=0
$$

The roots of this equation give us the characteristic values $\lambda=\lambda_{i}$, whereas the solution (24) yields the characteristic solutions, or eigenvalues, or principal axes of the matrix $A$ :

$u_{i}=S_{m-1}\left(\lambda_{i}\right) b_{0}+S_{m-2}\left(\lambda_{i}\right) \lambda_{i} b_{1}+\ldots+S_{0} \lambda_{i}^{m-1} b_{m-1}$.

It is a remarkable fact that although the vector $b_{0}$ was chosen entirely freely, the particular linear combination (27) of the iterated vectors has invariant significance, except for an undetermined factor of proportionality that remains frec, in view of the linearity of the defining equation (25). That undetermined factor may come out even as zero, i. c., a certain axis may not be represented in the trial vector $b_{0}$ at all. This explains why the order of the polynomial $S_{m}(\lambda)$ need not be necessarily equal to $n$. The trial vector $b_{0}$ may not give us all the principal axes of $A$. What we can say with assurance, however, is that all the roots of $S_{m}(\lambda)$ are true characteristic values of $A$, and all the $u_{1}$ obtained by the formula (24) are true characteristic vectors, even if we did not-obtain the complete solution of the eigenvalue problem. The discrepancy between the order $m$ of the polynomial $G(\mu)$ and the order $n$ of the characteristic equation

$$
F(\mu)=\left|\begin{array}{lll}
a_{11}-\mu & \cdots & a_{n n} \\
\cdot & \\
a_{n l} & \cdot a_{n n}-\mu
\end{array}\right|=0
$$

will be the subject of the discussion in the next section.

Instead of substituting into the formula (27), we can also obtain the principal axes $u_{i}$ by a numerically simpler process, applying synthetic division. By synthetic division we generate the polynomials :

$$
\frac{G(x)}{x-\mu_{i}}=x^{m-1}+g_{1}^{i} x^{m-2}+\ldots+g_{m-1}^{i} .
$$

We then replace $x^{5}$ by $b_{3}$ and obtain:

$$
u_{i}=b_{m-1}+g_{1}^{i} b_{m-2}+\ldots+g_{m-1}^{i} b_{0} .
$$

The proof follows immediately from the equation

$$
\left(A-\mu_{t}\right) u_{i}-G(A) \cdot b_{0}=0 .
$$

\section{The Problem of Missing Axes}

Let us assume that we start with an arbitrary "trial vector" $b_{0}$ and obtain by successive iterations the sequence:

$$
b_{0}, b_{1}, b_{2}, \ldots, b_{n} .
$$

Similarly we start with the trial vector $b_{0}^{*}$ and obtain by iterating with the transposed matrix $A^{*}$ the adjoint sequence:

$$
b_{0}^{*}, b_{1}^{*}, b_{2}^{*}, \ldots, b_{n}^{*} \text {. }
$$

Let us now form the following set of "basic scalars":

$$
c_{i+k}=b_{i} \cdot b_{k}^{*}=b_{k} \cdot b_{i}^{*} \text {. }
$$

The remarkable fact holds that these scalars depend only on the sum of the two subscripts $i$ and $k$; e. g.

$$
b_{k-1} b_{k+1}^{*}=b_{k-1}^{*} b_{k+1}=b_{k} b_{k}^{*}
$$

This gives a powerful numerical check of the iteration scheme since a discrepancy between the two sides of (35) (beyond the limits of the rounding errors) would indicate an error in the calculation of $b_{k+1}$ or $b_{k+1}^{*}$ if the scquence up to $b_{k}$ and $b_{k}^{*}$ has been checked before.

Let us assume for the sake of the present argument that $A$ is a nondefective matrix, and let us analyze the vector $b_{0}$ in terms of the eigenvectors $u_{i}$, while $b_{0}^{*}$ will be analyzed in terms of the adjoint vectors $u_{i}^{*}$ :

$$
\begin{aligned}
& b_{\mathrm{b}}=\tau_{1} u_{1}+\tau_{2} u_{2}+\ldots+\tau_{n} u_{n}, \\
& b_{0}^{*}=\tau_{1}^{*} u_{1}^{*}+\tau_{2}^{*} u_{2}^{*}+\ldots+\tau_{n}^{*} u_{n}^{*} .
\end{aligned}
$$


Then the scalars $c_{t}$ become:

with

$$
c_{i}=\rho_{1} \mu_{1}^{i}+\rho_{2} \mu_{2}^{i}+\ldots+\rho_{n} \mu_{n}^{i},
$$

$$
\rho_{k}=\tau_{k} r_{k}^{*} .
$$

The problem of obtaining the $\mu_{i}$ from the $c_{i}$ is the problem of "weighted moments", which can be solved as follows: Assuming that none of the $\rho_{k}$ vanish and that all the $\lambda_{i}$ are distinct, we establish a linear relation between $n+1$ consecutive $c_{i}$, of the following form:

$$
\left.\begin{array}{l}
c_{0} \eta_{0}+c_{1} \eta_{1}+\ldots+c_{n-1} \eta_{n-1}+c_{n}=0 \\
c_{1} \eta_{0}+c_{2} \eta_{1}+\ldots+c_{n} \eta_{n-1}+c_{n+1}=0 \\
\vdots \\
c_{n} \eta_{0}+c_{n+1} \eta_{1}+\ldots+c_{2 n-1} \eta_{n-1}+c_{2 n}=0
\end{array}\right\}
$$

Then the definition of the $c_{i}$ shows directly that the set (40) demands

where

$$
F\left(\mu_{i}\right)=0,
$$

$$
F(x)=\eta_{0}+\eta_{1} x+\ldots+\eta_{n-1} x^{n-1}+x^{n} .
$$

Hence, by solving the recurrent set (40) with the help of a "progressive algorithm", displayed in the next section, we can obtain the cocfficients of the characteristic polynomial (42), whose roots give the eigenvalues $\mu_{i}$.

Under the given restricting conditions none of the $\mu_{t}$ roots has been lost, and we could actually establish the full characteristic equation (41). It can happen, however, that $b_{0}$ is orthogonal to some axis $u_{i}^{*}$, and it is equally possible that $b_{0}^{*}$ is orthogonal to some axis $u_{k}$. In that case $r_{i}$ and $\tau_{k}^{*}$ drop out of the expansions (36) and (37) and consequently the expansion (38) lacks both $\rho_{t}$ and $\rho_{k}$. This means that the scalars $c_{j}$ are unable to provide all the $\mu_{y}$, since $\mu_{i}$ and $\mu_{k}$ are missing. The characteristic equation (41) cannot be fully established under these circumstances.

The deficiency was here caused by an unsuitable choice of the vectors $b_{0}$ and $b_{0}^{*}$; it is removable by a better choice of the trial vectors. However, we can have another situation where the deficiency goes deeper and is not removable by any choice of the trial vectors. This happens if the $\mu_{i}$ roots of the characteristic equation are not all distinct. The expansion (38) shows that two equal roots $\lambda_{t}$ and $\lambda_{k}$ cannot be separated since they behave exactly as one single root with a double amplitude. Generally, the weighted moments $c_{i}$ can never show whether or not therc arc multiple roots, because the multiple roots behave like single roots. Consequently, in the case of multiple eigenvalues the linear relation between the $c_{i}$ will not be of the $n^{\text {th }}$ but of a lower order. If the number of distinct roots is $m$, then the relation (40) will appear in the following form:

$$
\left.\begin{array}{l}
c_{0} \eta_{0}+c_{1} \eta_{1}+\ldots+c_{m-1} \eta_{m-1}+c_{m}=0 \\
c_{1} \eta_{0}+c_{2} \eta_{1}+\ldots+c_{m} \eta_{m-1}+c_{m+1}=0 \\
. \\
c_{m} \eta_{0}+c_{m+1} \eta_{1}+\ldots+c_{2 m-1} \eta_{m-1}+c_{2 m}=0
\end{array}\right\}
$$

Once more we can establish the polynomial

$$
G(x)=\eta_{0}+\eta_{1} x+\ldots+\eta_{m-1} x^{m-1}+x^{m},
$$

but this polynomial is now of only $m^{t h}$ order and factors into the $m$ root factors

$$
\left(x-\mu_{1}\right)\left(x-\mu_{2}\right) \ldots\left(x-\mu_{m}\right),
$$

where all the $\mu_{i}$ are distinct. After obtaining all the roots of the polynomial (44) we can now construct by synthetie division the polynomials:

$$
\frac{G(x)}{r-\mu_{k}}=x^{m-1}+g_{1}^{k} x^{m-2}+\ldots+g_{m-1}^{k},
$$

and replacing $x^{j}$ by $b_{f}$ we obtain the principal axes of both $A$ and $A^{*}$ :

$$
\left.\begin{array}{l}
u_{k}=b_{m-1}+g_{1}^{k} b_{m-2}+\ldots+g_{m-1}^{k} b_{0}, \\
u_{k}^{*}=b_{m-1}^{*}+g_{1}^{k} b_{n-2}^{*}+\ldots+g_{m-1}^{k} b_{0}^{*} .
\end{array}\right\}
$$

This gives a partial solution of the prineipal axis problem, inasmuch as each multiple root contributed only one axis. Moreover, we cannot tell from our solution which one of the roots is single and which one multiple, nor can the degree of multiplicity be established. In order to get further information, we have to change our trial vectors and go through the iteration scheme once more. We now substitute into the formulae (47) again and can immediately localize all the single roots by the fact that the vectors $u_{f}$ associated with these roots do not change (apart from a proportionality factor), whereas the $u_{k}$ belonging to double roots will generally change their direction. 
A proper linear combination of the new $u_{k}^{*}$, and the previous $u_{k}$ establishes the second axis associated with the double eigenvalue $\mu_{k}$; we put

$$
\begin{array}{cc}
u_{k}^{1}=u_{k} & u_{k}^{2}=u_{k}^{+}+\gamma u_{k} \\
u_{k}^{1 *}=u_{k}^{*} & u_{k}^{2^{* *}}=u_{k}^{*}+\gamma^{*} u_{k}^{*}
\end{array}
$$

The factors $\gamma$ and $\gamma^{*}$ are determined by the conditions that the vectors $u_{k}^{\mathrm{z}}$ and $u_{k}^{2}$ have to be biorthogonal to the vectors $u_{k}^{1^{*}}$ and $u_{k}^{2^{*}}$. In the case of triple roots a third trial is demanded, and so on.

An interesting contrast to this behavior of multiple roots associated with nondefective matrices is provided by the behavior of multiple roots associated with defective matrices. A defective eigenvalue is always a multiple eigenvalue, but here the multiplicity is not caused by the collapse of two very near eigenvalues, but by the multiplicity of the elementary divisor. This comes into evidence in the polynomial $G(x)$ by giving a root factor of higher than first order. Whenever the polynomial $G(x)$ reveals a multiple root, we can tell in advance that the matrix $A$ is defective in these roots, and the multiplicity of the root establishes the degree of deficiency.

It will be revealing to demonstrate these conditions with the help of a matrix that combines all the different types of irregularities that may be encountered in working with arbitrary matrices. Let us analyze the following matrix of sixth order:

$$
\left(\begin{array}{llllll}
1 & 2 & 3 & 0 & 0 & 0 \\
0 & 1 & 4 & 0 & 0 & 0 \\
0 & 0 & 1 & 0 & 0 & 0 \\
0 & 0 & 0 & 2 & 0 & 0 \\
0 & 0 & 0 & 0 & 0 & 0 \\
0 & 0 & 0 & 0 & 0 & 0
\end{array}\right)
$$

The eigenvalue 2 is the only regular eigenvalue of this matrix. The matrix is "singular", because the determinant of the coefficients is zero. This, however, is irrelevant from the viewpoint of the eigenvalue problem, since the eigenvalue "zero" is just as good as any other eigenvalue. More important is the fact that the eigenvalue zero is a double root of the characteristic equation. The remaining three roots of the characteristic equation are all 1. This 1 is thus a triple root of the characteristic equation; at the same time the matrix has a double deficiency in this root, because the elementary divisor associated with this root is cubic. The matrix possesses only four independent principal axes.

What will the polynomial $G(x)$ become in the case of this matrix? The regular eigenvalue 2 must give the root factor $x-2$. The regular eigenvalue 0 has the multiplicity 2 but is reduced to the single eigenvalue 0 and thus contributes the factor $x$. The deficient eigenvalue 1 has the multiplicity 3 but also double defectiveness. Hence, it must contribute the root factor $(x-1)^{3}$. We can thus predict that the polynomial $G(x)$ will come out as follows.

$$
G(x)=x(x-2)(x-1)^{3}=x^{5}-5 x^{4}+9 x^{3}-7 x^{2}+2 x .
$$

Ist us verify this numerically. As a trial vector we choose

$$
b_{0}=b_{0}^{*}=1,1,1,1,1,1 \text {. }
$$

The successive iterations yield the following:

$\begin{array}{rrrrrrr}b_{0}= & 1 & 1 & 1 & 1 & 1 & 1 \\ b_{1}= & 6 & 5 & 1 & 2 & 0 & 0 \\ b_{2}= & 19 & 9 & 1 & 4 & 0 & 0 \\ b_{3}=40 & 13 & 1 & 8 & 0 & 0 \\ b_{4}=69 & 17 & 1 & 16 & 0 & 0 \\ b_{5}=106 & 21 & 1 & 32 & 0 & 0 \\ b_{6}=151 & 25 & 1 & 64 & 0 & 0 \\ b_{0}^{*}= & 1 & 1 & 1 & 1 & 1 & 1 \\ b_{1}^{*}= & 1 & 3 & 8 & 2 & 0 & 0 \\ b_{2}^{*}= & 1 & 5 & 23 & 4 & 0 & 0 \\ b_{3}^{*}= & 1 & 7 & 46 & 8 & 0 & 0 \\ b_{4}^{*}= & 1 & 9 & 77 & 16 & 0 & 0 \\ b_{5}^{*}= & 1 & 11 & 116 & 32 & 0 & 0 \\ b_{8}^{*}= & 1 & 13 & 163 & 64 & 0 & 0\end{array}$

We now construct the $c_{1}$ by dotting $b_{0}$ with the $b_{i}^{*}$ (or $b_{0}^{*}$ with the $b_{i}$ ); we continue by dotting $b_{6}$ with $b_{1}^{*}, \ldots b_{6}^{*}$ (or $b_{0}^{*}$ with $b_{1}, \ldots b_{6}$ ). This gives . the following set of $2 n+1=13$ basic scalars.

$c_{j}=6,14,33,62,103,160,241,362,555,884$, $1477,2590,4735$. 
The application of the progressive algorithm of section VI to these $b_{i}$ yields $G(x)$ in the predicted form. Wo now obtain by synthetic divisions:

$$
\begin{aligned}
& \frac{G(x)}{x-1}=x^{4}-4 x^{3}+5 x^{2}-2 x \\
& \frac{G(x)}{x-2}=x^{4}-3 x^{3}+3 x^{2}-x, \\
& \frac{G(x)}{x}=x^{4}-5 x^{4}+9 x^{2}-7 x+2 .
\end{aligned}
$$

Inverting these polynomials we obtain the matrix

$$
\left(\begin{array}{llllll}
0 & -2 & 5 & -4 & 1 & 0 \\
0 & -1 & 3 & -3 & 1 & 0 \\
2 & -7 & 9 & -5 & 1 & 0
\end{array}\right)
$$

The product of this matrix with the iteration matrix $B$ (omitting the last row $b_{b}$ ) yields three principal axes $u_{i}$; similarly the product of the same matrix with the iteration matrix $B^{*}$ yields the three adjoint axes $u_{i}^{*}$ :

$$
\begin{array}{ccccccc}
u(1)=-8 & 0 & 0 & 0 & 0 & 0 \\
u(2)= & 0 & 0 & 0 & 2 & 0 & 0 \\
u(0)= & 0 & 0 & 0 & 0 & 2 & 2 \\
u^{*}(1)= & 0 & 0-8 & 0 & 0 & 0 \\
u^{*}(2)= & 0 & 0 & 0 & 2 & 0 & 0 \\
u^{*}(0)= & 0 & 0 & 0 & 0 & 2 & 2
\end{array}
$$

Since $G(x)$ is of only fifth order, while the order of the characteristic equation is 6 , we know that one of the axes is still missing. We cannot decide a priori whether the missing axis is caused by the duplicity of the eigenvalue 0,1 , or $2 .^{9}$ However, a repetition of the iteration with the trial vectors

$$
b_{0}=b_{0}^{*}=1,1,1,1,1,0,
$$

causes a change in the row $u(0)$ and $u^{*}(0)$ only. This designates the eigenvalue $u=0$ as the double root. The process of biorthogonalization finally yields: ${ }^{\text {I0 }}$

\footnotetext{
- We have in mind the general case and overlook the fact that tho oversimplified nature of the $€$ xample makes the decision trivial.

20 The reader is urged to earry through a similar anulysis with the same matrix, but changing the 0,0 diagonal elements of the rows 5 and $B$ to 1,0 , and to 1,1 .
}

$$
\begin{array}{rllllr}
u_{1}(0)=0 & 0 & 0 & 0 & 1 & 1 \\
u_{2}(0)=0 & 0 & 0 & 0 & 1 & -1 \\
u_{1}^{*}(0)=0 & 0 & 0 & 0 & 1 & 1 \\
u_{2}^{*}(0)=0 & 0 & 0 & 0 & 1 & -1
\end{array}
$$

\section{The Progressive Algorithm for the Construction of the Characteristic Poly- nomial $\mathrm{G}(\mathrm{x})$}

The crucial point in our discussions was the establishment of a linear relation between a certain $b_{m}$ and the previous iterated vectors. This ratation leads to the characteristic polynominal $G(x)$, whose roots $G\left(\mu_{i}\right)=0$ yield the eigenvalues $\mu_{1}$. Then by synthetic division we can immediately obtain those particular linear combinations of the iterated vectors $b_{i}$, which give us the eigenvectors (principal axes) of the matrix $A$.

We do not know in advance in what relation the order $m$ of the polynomial $G(x)$ will be to the order $n$ of the matrix $A$. Accidental deficiencies of the trial vectors $b_{0}, b_{0}^{*}$, and the presence of multiple eigenvalues in $A$ can diminish $m$ to any value between 1 and $n$. For this reason we will follow a systematic procedure that generates $G(x)$ gradually, going through all degrees from 1 to $m$. The procedure comes automatically to a halt when the proper $m$ has been reached.

Our final goal is to solve the recurrent set of equations:

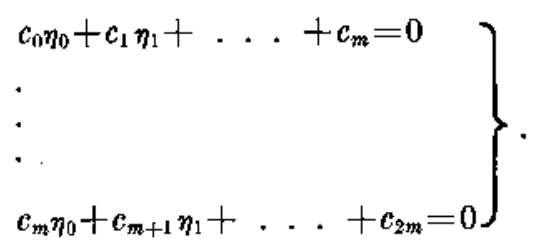

This is only possible if the determinant of this homogeneous set vanishes:

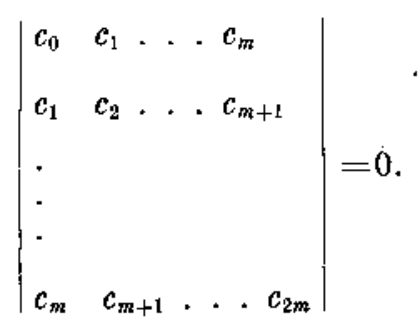


Before reaching this goal, however, we can certainly solve for any $k<m$ the following inhomogeneous set (the upper $k$ is meant as a superscript):

$$
\left.\begin{array}{l}
c_{0} \eta_{0}^{k}+c_{1} \eta_{1}^{k}+\ldots+c_{k}=0 \\
c_{1} \eta_{0}^{k}+c_{2} \eta_{1}^{k}+\ldots+c_{k+1}=0 \\
\cdot \\
c_{k} \eta_{0}^{k}+c_{k+1} \eta_{1}^{k}+\ldots+c_{2 k}=h_{k}
\end{array}\right\}
$$

The freedom of $h_{k}$ in the last equation removes the overdetermination of the set (48). The proper $m$ will be reached as soon as $h_{m}$ turns out to be zero.

Now a recurrent set of equations has certain algebraic properties that are not shared by other linear systems. In particular, there exists a recurrence relation between the solutions of three consecutive sets of the type (50). This greatly facilitates the method of solution, through the application of a systematic recurrence scheme that will now be developed.

We consider the system (50) and assume that we possess the solution up to a definite $k$. Then we will show how this solution may be utilized for the construction of the next solution, which belongs to the order $k+1$.

Our scheme becomes greatly simplified if we consider an additional set of equations that omits the first equation of (50) but adds one more equation at the end:

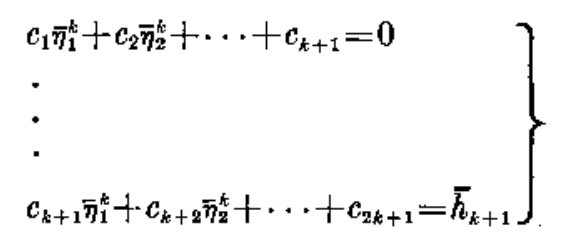

Let us now multiply the set (50) by the factor

$$
q_{k}=-\frac{\vec{h}_{k+1}}{h_{k}},
$$

and add the set (51). We get a new set of equations that can be written down in the form:

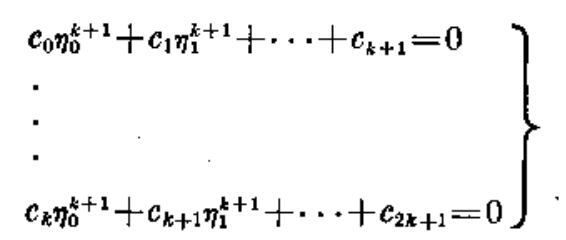

provided that we put

$$
\left.\begin{array}{l}
\eta_{0}^{k+1}=q_{k} \eta_{0}^{k} \\
\eta_{1}^{k+1}=q_{k} \eta_{1}^{k}+\bar{\eta}_{1}^{k} \\
\cdot \\
\eta_{k}^{k+1}=q_{k} \cdot 1+\bar{\eta}_{k}^{k}
\end{array}\right\}
$$

We now evaluate the scalar

$$
c_{k+1} \eta_{0}^{k+1}+\cdots+c_{2 k+1} \eta_{k}^{k+1}+c_{2 k+2}=h_{k+1}
$$

which is added to (53) as the last equation.

What we have accomplished is that a proper linear combination of the solutions $\eta_{i}^{k}$ and $\bar{\eta}_{i}^{k}$ provided us with the next solution $\eta_{i}^{k+1}$. But now exactly the same procedure can be utilized to obtain $\bar{\eta}_{i}^{k+1}$ on the basis of $\bar{\eta}_{i}^{k}$ and $\eta_{i}^{k+1}$.

For this purpose we multiply the set (51) by

$$
\bar{q}_{k}=-\frac{h_{k+1}}{\bar{h}_{k+1}}
$$

and add the set (53), completed by (55) but omitting the first equation.

This gives:

$$
\left.\begin{array}{l}
c_{1} \bar{\eta}_{1}{ }^{k+1}+c_{2} \bar{\eta}_{2}^{k+1}+\cdots+c_{k+2}=0 \\
\cdot \\
c_{k+1} \bar{\eta}_{1}^{k+1}+c_{k+2} \bar{\eta}_{2}^{k+1}+\cdots+c_{2 k+2}=0
\end{array}\right\}
$$

provided that we put:

$$
\left.\begin{array}{l}
\bar{\eta}_{1}^{k+1}=\bar{q}_{k} \bar{\eta}_{1}^{k}+\eta_{0}^{k+1} \\
\bar{\eta}_{2}^{k+1}=\bar{q}_{k} \bar{\eta}_{2}^{k}+\eta_{1}^{k+1} \\
\cdot \\
\cdot \\
\bar{\eta}_{k+1}^{k+1}=\bar{q}_{k} \cdot 1+\eta_{k}^{k+1}
\end{array}\right\}
$$

Once more we evaluate the scalar

$$
c_{k+2} \bar{\eta}_{1}^{k+1}+\cdots+c_{2 k+3}=\bar{h}_{k+2},
$$

which is added to (57) as the last equation.

This analytical procedure can be translated into an elegant geometrical arrangcment that generates the successive solutions $\eta_{i}^{k}$ and $\bar{\eta}_{i}^{k}$ in successive columns. The resulting algorithm is best explained with the help of a numerical example.

For this purpose we choose the eigenvalue problem of an intentionally over-simplified matrix, since our aim is not to show the power of the 
method but the nature of the algorithm, which leads to the establishment of the characteristic equation. The limitation of the method due to the accumulation of rounding errors will be discussed in the next section.

Let the given matrix be:

$$
\left(\begin{array}{rrr}
13 & 5 & -23 \\
4 & 0 & -4 \\
7 & 3 & -13
\end{array}\right)
$$

We iterate with the trial vector $b_{0}=1,0,0$, and obtain:

$\begin{array}{rrr}1 & 0 & 0 \\ 13 & 4 & 7 \\ 28 & 24 & 12 \\ 208 & 64 & 112\end{array}$

We transpose the matrix and iterate with the trial vector $b_{0}{ }^{*}=1,0,0$, obtaining:

$\begin{array}{rrr}1 & 0 & 0 \\ 13 & 5 & -23 \\ 28 & -4 & -20 \\ 208 & 80 & -368\end{array}$

We dot the first row and the last row with the opposing matrix and obtain the basic scalars $c_{i}$ as follows: ${ }^{\text {it }}$

$1,13,28,208,448,3328,7168$.

These numbers are written down in a column, and tho scheme displayed below is obtained.

\begin{tabular}{|c|c|c|c|c|c|c|c|}
\hline & 0 & 0.5 & 1 & 1.5 & 2 & $\varepsilon, 5$ & 3 \\
\hline$h_{i}=$ & 1 & 13 & -141 & 147,6923075 & -163.4012569 & 0 & 0 \\
\hline$q^{\prime}=$ & -13 & 10. 84615384 & 1. 047483173 & 1. 106382990 & 0 & & \\
\hline$t$ & 1 & & & & & & \\
\hline 13 & & 1 & -13 & & & & \\
\hline 28 & & & 1 & -2.15384616 & -13.617021249 & & \\
\hline 208 & & & & 1 & $-1.10638208 \pi$ & -1 B. 000000004 & 0 \\
\hline 418 & & & & & 1 & 0.000000003 & -18 \\
\hline 3328 & & & & & & I & 0 \\
\hline 7138 & & & & & & & 1 \\
\hline
\end{tabular}

Instead of distinguishing between the $\eta_{i}$ and $\bar{\eta}_{i}$ solutions we use a uniform procedure but mark the successive columns alternately as "full" and "half columns"; thus we number the successive columns as zero, one-half, one, . . The scheme has to end at a full column, and the end is marked by the vanishing of the corresponding "headnumber" $h_{\text {f. }}$ In our scheme the head-number is zero already at the half-column 2.5, but the scheme cannot end here, and thus we continue to the column 3, whose head-number becomes once more 0 , and then the scheme is finished. The last column gives the polynomial $G(x)$, starting with the diagonal term and proceeding upward:

$$
\begin{aligned}
G(x) & =1 \cdot x^{3}+0 \cdot x^{2}-16 x+0 \\
& =x^{3}-16 x
\end{aligned}
$$

The head-numbers $h_{t}$ are always obtained by dotting the column below with the basic column $c_{i}$; e. g., at the head of the column 2 we find the number -163.4042569. This number was obtained by the following cumulative multiplication:

$448 \cdot 1+208 \cdot(-1.106382987)+28 \cdot(-13.617021249)$.

The numbers $q_{i}$ represent the negative ratio of two consecutive $h_{i}$ numbers:

$$
q_{i}=-\frac{h_{i+1 / i}}{h_{i}}
$$

\footnotetext{
11 Instead of iterating with $A$ and $A^{*} n$ times, we can also iterste with $A$ gione $2 n$ times. Any of the collumns of the iteration matrlx can now be chosen as $a$ numbers since these colnmns correspond to a dotting of the fteration matrix with $b_{0}^{*}=1,0,0, \ldots$, respectively $0,1,0,0, \ldots ; 0,0,1,0$, $0, \ldots ;$ and so ov. The transpased matrix is not used here at all, E. C. Bower of the Douglas Aireraft Co. points out to the author that from the machine viewpoint a uniform iteration seheme of $2 n$ iterstlons is preferable to a divided scheme of $n+n$ iterations. The divided scheme has the advantage of less accumulation of rounding errors and more powerful checks on the successive iterations. The uniform soheme has the advantage that more than one colutmn is at our disposal. Accidentul defleiencies of the $0_{0}^{*}$ vector can thus be eliminated, by repeating the algorithm with a different column. (For this purpase it is of advantage to start with the trial vector $b_{0}=1,1$, $1, \ldots .1$.) In the case of a symmetric matrix it is evident that after $n$ itteratlons the basic sealars should be formed, Instead of continuing with $n$ more tterations.
} 
e. g., $q_{1 . s}=1.106382990$ was obtained by the following division:

$$
\frac{-(-163.4042569)}{147.6923075}
$$

The scheme grows as follows. As soon as a certain column $C_{t}$ is coinpleted, we evaluate the associated head-number $h_{i}$; this provides us with the previous $q$-number $\dot{q}_{i-3}$. We now construct. the next column $C_{i+1 / 4}$ by the following operation. We multiply the column $C_{i-k}$ by the constant $q_{i-3 / 6}$ and add the column $C_{i}$ :

$$
C_{i+3}=q_{i-3,} \cdot C_{i-34}+C_{i}
$$

However, the result of this operation is shifted down by one element; e. g. in constructing the column 2.5 the result of the operation

$$
\begin{aligned}
& 1.10638299 \cdot(-2.15384616)+ \\
& (-13.617021249)=-16,
\end{aligned}
$$

is not put in the row where the operation occurred, but shifted down to the next row below.

The unfilled spaces of the scheme are all "zero".

The outstanding feature of this algorithm is that it can never come to premature grief, provided only that the first two $c$-numbers, $c_{0}$ and $c_{1}$, are different from zero. Division by zero cannot occur since the scheme comes to an end anyway as soon as the head-number zero appears in one of the full columns.

Also of interest is the fact that the products of the head-numbers associated with the full columns give us the successive recurrent determinants of the $c_{i} ;$ e.g., the determinants

$$
\begin{array}{r|rr}
1 & 1 & 13 \\
13 & 28
\end{array}|\quad| \begin{array}{rrr}
1 & 13 & 28 \\
13 & 28 & 208 \\
28 & 208 & 448
\end{array}
$$

and

\begin{tabular}{|rrrr}
1 & 13 & 28 & 208 \\
13 & 28 & 208 & 448 \\
28 & 208 & 448 & 3328 \\
208 & 448 & 3328 & 7168
\end{tabular}

are given by the successive products

$1,1 \cdot(-141)=-141,(-141) \cdot(-163.4042569)=$ 23040 , and $23040 \cdot 0=0$,
Similarly the products of the head-numbers of the half-columns give us similar determinants, but omitting $c_{0}$ from the sequence of $c$-numbers. In the example above the determinants

13 ,

$$
\left|\begin{array}{rr}
13 & 28 \\
28 & 208
\end{array}\right| \cdot\left|\begin{array}{rrr}
13 & 28 & 208 \\
28 & 208 & 448 \\
208 & 448 & 3328
\end{array}\right|
$$

are given by the products:

$$
13,13 \cdot 147.6923075=1920,1920 \cdot 0=0
$$

The purpose of the algorithm (60) was to genera te the coefficients of the basic identity that exists between the iterated vectors $b_{i}$. This identity finds expression in the vanishing of the polynomial $G(x)$ :

$$
G(x)=0
$$

The roots of this algebraic equation give us the eigenvalues of the matrix $A$. In our example we get the cubic equation

$$
x^{3}-16 x=0,
$$

which has the three roots

$$
\mu_{1}=0, \mu_{2}=4, \mu_{3}=-4 \text {. }
$$

These are the eigenvalues of our matrix. In order to obtain the associated eigenvectors, we divide $G(x)$ by the root factors:

$$
\frac{G(x)}{x}=x^{2}-16 \quad \frac{G(x)}{x-4}=x^{2}+4 x \quad \frac{G(x)}{x+4}=x^{2}-4 x .
$$

This gives, replacing $x^{k}$ by $b_{k}$ :

$$
\begin{aligned}
& u(0)=-16 b_{0}+b_{2}, \\
& u(4)=4 b_{1}+b_{2},
\end{aligned}
$$

$$
u(-4)=-4 b_{1}+b_{2} \text {. }
$$

Consequently, if the matrix

$$
\left(\begin{array}{rrr}
-16 & 0 & 1 \\
0 & 4 & 1 \\
0 & -4 & 1
\end{array}\right)
$$

is multiplied by the matrix of the $b_{i}$ (omitting $b_{3}$ ), we obtain the three eigenvectors $u_{i}$ : 


$$
\left(\begin{array}{rrr}
-16 & 0 & 1 \\
0 & 4 & 1 \\
0 & -4 & 1
\end{array}\right) \cdot\left(\begin{array}{rrr}
1 & 0 & 0 \\
13 & 4 & 7 \\
28 & 24 & 12
\end{array}\right)=\left(\begin{array}{rrc}
12 & 24 & 12 \\
80 & 40 & 40 \\
-24 & 8 & -16
\end{array}\right)=\left(\begin{array}{l}
u(0) \\
u(4) \\
u(-4)
\end{array}\right)
$$

If the same matrix is multiplied by the matrix of the transposed iterations $b_{1}^{*}$ (omitting $b_{3}^{*}$ ), we obtain the three adjoint eigenvectors $u_{i}^{*}$ :

$$
\left(\begin{array}{rrr}
-16 & 0 & 1 \\
0 & 4 & 1 \\
0 & -4 & 1
\end{array}\right) \cdot\left(\begin{array}{rrr}
1 & 0 & 0 \\
13 & 5 & -23 \\
28 & -4 & -20
\end{array}\right)=\left(\begin{array}{rrr}
12 & -4 & -20 \\
80 & 16 & -112 \\
-24 & -24 & 72
\end{array}\right)=\left(\begin{array}{l}
u^{*}(0) \\
u^{*}(4) \\
u^{*}(-4)
\end{array}\right)
$$

The solution of the entire eigenvalue problem is thus accomplished.

\section{The Method of Minimized Iterations}

In principle, the previous discussions give a complete solution of the eigenvalue problem. We have found a systematic algorithm for the generation of the characteristic polynomial $G(\mu)$. The roots of this polynomial gave the eigenvalues of the matrix $A$. Then the process of synthetic division established the associated eigenvectors. Accidental deficiencics were possible but could be eliminated by additional trials.

As a matter of fact, however, the "progressive algorithm" of the last section has its serious limitations if large matrices are involved. Let us assume that there is considerable "dispersion" among the eigenvalues, which means that the ratio of the largest to the smallest eigenvalue is fairly large. Then the successive iterations will grossly increase the gap, and after a fow iterations the small eigenvalues will be practically drowned out. Let us assume, e. g., that we have a 12 by 12 matrix, which requires 12 iterations for the generation of the characteristic equation. The relatively mild ratio of $10: 1$ as the "spread" of the eigenvalues is after 12 iterations increased to the ratio $10^{\mathrm{I} 2}: 1$, which means that we can never get through with the iteration scheme because the rounding errors make all iterations beyond the eighth entirely valueless.

As an actual example, taken from a physical situation, let us consider four eigenvalues, which are distributed as follows:

$$
1,5,50,2000 .
$$

Let us assume, furthermore, that we start with a trial vector that contains the four eigenvectors in the ratio of the eigenvalues, $i$. e., the eigenvalue 2000 dominates with the amplitude 2000 , compared with the amplitude of the eigenvalue 1. After one iteration the amplitude ratio is increased to $4 \cdot 10^{8}$, after two iterations to $8 \cdot 10^{\circ}$. The later iterations can give us no new information, since they practically repeat the second iteration, multiplied every time by the factor 2000 . The small eigenvalues 1 and 5 are practically obliterated and cannot be rescued, except by an excessive accuracy that is far beyond the limitations of the customary digital machines.

We will now develop a modification of the customary iteration technique that dispenses with this difficulty. The modified scheme eliminates the rapid accumulation of rounding errors, which under ordinary circumstances destroys the value of high order iterations. The new technique prevents the large cigenvalues from monopolizing the scenc. It protects the small cigenvalues by constantly balancing the distribution of amplitudes in the most equitable fashion.

As an illustrative example, let us apply this method of "minimized iterations" to the abovementioned dispersion problem. If the largest amplitude is normalized to 1 , then the initial distribution of amplitudes is characterized as follows:

$$
0.0005, \quad 0.0025, \quad 0.025, \quad 1 \text {. }
$$

Now, while an ordinary iteration would make this distribution still more extreme, the method of minimized iterations changes the distribution of amplitudes as follows:

$$
\begin{array}{lllll}
0.0205 & 0.1023 & 1 & -0.0253
\end{array}
$$

We see that it is now the third eigenvector that gets a large weight factor, whereas the fourth eigenvector is almost completely in the background.

A repetition of the scheme brings about the following new distribution:

$$
\begin{array}{llll}
0.2184 & 1 & -0.1068 & 0.0000 .
\end{array}
$$


It is now the second eigenvector that gets the strongest emphasis.

The noxt repetition yields:

$$
1 \quad-0.2181 \quad 0.0018 \quad 0.0000,
$$

and we see that the weight shifted over to the smallest eigenvalue.

After giving a chance to each eigenvalue, the scheme is exhausted, since we have all the information we need. Consequently the next minimized iteration yields an identical vanishing of the next vector, thus bringing the scheme to its natural conclusion.

In order to expose the principle of minimized iterations, let us first consider the case of symmetric matrices:

$$
A^{*}=A
$$

Moreover, let us agree that the multiplication of a vector $b$ by the matrix $A$ shall be denoted by a prime:

$$
A b=b^{\prime}
$$

Now our aim is to establish a linear identity between the iterated vectors. We cannot expect that this identity will come into being right from the beginning. Yet we can approach this identity right from the beginning by choosing that linear combination of the iterated vectors $b_{0}^{\prime}$ and $b_{0}$ which makes the amplitude of the new vector as small as possible. Hence, we want to choose as our new vector $b_{1}$ the following combination:

$$
b_{1}=b_{0}^{\prime}-\alpha_{0} b_{0}
$$

where $\alpha_{0}$ is determined by the condition that

This gives

$$
\left\langle b_{o}^{\prime}-\alpha_{0} b_{0}\right)^{2}=\text { minimum } .
$$

$$
\alpha_{0}=\frac{b_{0}^{\prime} b_{0}}{b_{0}^{2}}
$$

Notice that

$$
b_{1} \cdot b_{0}=0
$$

i.e. the new vector $b_{1}$ is orthogonal to the original vector $b_{0}$.

We now continue our process. From $b_{1}$ we proceed to $b_{2}$ by choosing the linear combination

$$
b_{2}=b_{1}^{\prime}-\alpha_{1} b_{1}-\beta_{0} b_{0},
$$

and once more $\alpha_{1}$ and $\beta_{0}$ are determined by the condition that $b_{2}^{2}$ shall become as small as possible. This gives

$$
\alpha_{1}=\frac{b_{1}^{\prime} b_{1}}{b_{1}^{2}} \quad \beta_{0}=\frac{b_{1}^{\prime} b_{0}}{b_{0}^{2}}
$$

A good check of the iteration $b_{1}^{\prime}$ is provided by the condition

$$
b_{1}^{\prime} b_{0}=b_{1} b_{0}^{\prime}=b_{1}^{2}
$$

Hence, the numerator of $\beta_{0}$ has to agree with the denominator of $a_{1}$.

The new vector $b_{2}$ is orthogonal to both $b_{0}$ and $b_{1}$.

This scheme can obviously be continued. The most remarkable feature of this successive minimization process is, however, that the best linear combination never includes more than three terms. If we form $b_{3}$, we would think that we should put

$$
b_{3}=b_{2}^{\prime}-\alpha_{2} b_{2}-\beta_{1} b_{1}-\gamma_{0} b_{0} .
$$

But actually, in view of the orthogonality of $b_{2}$ to the previous vectors, we get

$$
\gamma_{0}=\frac{b_{2}^{\prime} b_{0}}{b_{0}^{2}}=\frac{b_{2} b_{0}^{\prime}}{b_{0}^{2}}=0
$$

Hence, every new step of the minimization process reguires only two correction terms.

By this process a succession of orthogonal vectors is generated: $:^{12}$

$$
b_{0}, b_{1}, b_{2}, \ldots, b_{m-1} \text {, }
$$

until the identity relation becomes exact, which means that

$$
b_{m}=0 \text {. }
$$

If the matrix $A$ is not symmetric, then we modify our procedure as follows: We operate simultaneously with $A$ and $A^{*}$. The operations are the same as before, with the only difference that the dot products are always formed between two opposing vectors. The scheme is indicated as follows:

$$
\begin{gathered}
b_{0}=b_{0}^{\prime}-\alpha_{0} b_{0} \quad b_{1}^{*}=b_{0}^{* \prime}-\alpha_{0} b_{0}^{*} \\
\alpha_{0}=\frac{b_{0}^{\prime} b_{0}^{*}}{b_{0} b_{0}^{*}=}=\frac{b_{0}^{* \prime} b_{0}}{b_{0}^{*} b_{0}}
\end{gathered}
$$

12 The Ides of the successive orthogomalization of a sct of vectors was probably first employed by 0 . Bzssz, in connection with a determinant theorem of Hedsmard; ef. Math. es phys. lapok (in Hungarian) 19, 221 to 227 , (1910), The method found later numerous important applications. 


$$
\begin{gathered}
b_{2}=b_{1}^{\prime}-\alpha_{1} b_{1}-\beta_{0} b_{0} \quad b_{2}^{*}=b_{1}^{* \prime}-\alpha_{1} b_{1}^{*}-\beta_{0} b_{0}^{*} \\
\alpha_{1}=\frac{b_{1}^{\prime} b_{1}^{*}}{b_{1} b_{1}^{*}}=\frac{b_{1}^{* \prime} b_{1}}{\bar{b}_{1}^{*} b_{1}} \\
\beta_{0}=\frac{b_{1}^{\prime} b_{0}^{*}}{b_{0}} b_{0}^{*}=\frac{b_{1}^{*} b_{0}}{b_{0}^{*} \bar{b}}=\frac{b_{1}^{*} b_{1}}{b_{0}^{*} b_{0}} \\
b_{2}=b_{2}^{\prime}-\alpha_{2} b_{2}-\beta_{1} b_{1} \quad b_{3}^{*}=b_{2}^{*}-\alpha_{2} b_{2}^{*}-\beta_{1} b_{1}^{*} \text { etc. }
\end{gathered}
$$

Operationally the prime indicates multiplication by the matrix $A$. Hence, the succession of $b_{i}$ vectors represents in fact a suecessive set of polynomials. Replacing $A$ by the more familiar letter $x$, we have:

$$
\left.\begin{array}{l}
b_{0}=1 \cdot b_{0} \\
b_{1}=\left(x-\alpha_{0}\right) b_{0} \\
b_{2}=\left(x-\alpha_{1}\right) b_{1}-\beta_{0} b_{0} \\
b_{3}=\left(x-\alpha_{2}\right) b_{2}-\beta_{1} b_{1} \\
\cdots \cdots \\
b_{m}=\left(x-\alpha_{m-1}\right) b_{m-1}-\beta_{m-2} b_{m-2}=0
\end{array}\right\}
$$

This gradual generation of the characteristic polynomial $G(x)$ is in complete harmony with the procedure of the "progressive algorithm", discussed in section VI. In fact, the successive polynomials of the set (77) are identical with the polynomials found in the full columns of the progressive algorithm $(60)$. This explains the existence of the recurrence relation

$$
p_{m+1}(x)=\left(x-\alpha_{n}\right) p_{n}(x)-\beta_{n-1} p_{n-1}(x),
$$

without additional $\gamma, \delta, . \quad . \quad$ terms. The existence of such a relation is a characteristic feature of the recurrent set of equations that are at the basis of the entire development.

Although the new scheme goes basically through the same steps as the previously discussed "progressive algorithm", it is in an incomparably stronger position concerning rounding errors. Apart from the fact that the rounding errors do not accumulate, we can effectively counteract their influence by constantly checking the mutual orthogonality of the gradually evolving vectors $b_{i}$ and $b_{i}^{*}$. Any lack of orthogonality, caused by rounding errors, can immediately be corrected by the addition of a small correction term..$^{13}$ By this procedure the orthogonality of the generated

\footnotetext{
Ir See section IX, eq 9 .
}

vector system does not come gradually out of gear.

However, quite apart from the numerical advantages, the biorthogonality of the vectors $b_{i}$ and $b_{i}^{*}$ has further appeal because it imitates the behavior of the prineipal axes. This is analytically an eminently valuable fact that makes the transition from the iterated vectors to the principal axes a simple and strongly convergent process.

In order to see the method in actual operation, let us apply it to the simple example of section VI. Hore the matrix $A$ is of third order, and thus we have to construet the vectors $b_{0}, b_{1}, b_{2}, b_{3}$, and the corresponding adjoint vectors. We obtain the following results:

$$
\begin{aligned}
& b_{0}=1 \quad 0 \quad 0 \quad b_{0}^{*}=1 \quad 0 \quad 0 \\
& b_{0}^{\prime}=13 \quad 4 \quad 7 \quad b_{0}^{* \prime}=13 \quad 5 \quad-23 \\
& \alpha_{0}=\frac{1 \cdot 13}{1}=13 \\
& b_{1}=\quad \begin{array}{llllll}
0 & 4 & 7 & b_{1}^{*}= & 0 & 5
\end{array} \\
& b_{1}^{\prime}=-141 \quad-28 \quad-79 \quad b_{1}^{\circ}=-141 \quad-69 \quad 279 \\
& \alpha_{1}=\frac{1677}{-141} \quad \beta_{0}=\frac{-141}{1}=-141 \\
& =-11.89361702 \\
& b_{2}=0,19.57446808,4.25531914 \\
& b_{2}^{*}=0,-9.53191490,5.44680854 \\
& b_{2}^{\prime}=0,-17.02127656,3.40425542 \\
& b_{2}^{* \prime}=0,16.34042562,-32.68085142 \\
& \alpha_{2}=\frac{180.78768715}{-163.4042553} \quad \beta_{1}=\frac{-163.40425746}{-141} \\
& =-1.106382981 \quad=1.158895443 \\
& b_{3}=0, \quad 0, \quad 0 \quad b_{3}^{*}=0, \quad 0, \quad 0
\end{aligned}
$$

The associated polynomials become:

$$
\begin{aligned}
p_{0}= & 1 \\
p_{1}(x)= & x-13 \\
p_{2}(x)= & (x+11.89361702)(x-13)+141 \\
= & x^{2}-1.10638298 x-13.61702126 \\
p_{3}(x)= & (x+1.106382981)\left(x^{2}+10638298 x-\right. \\
& 13.61702126)-1.158895443(x-13) \\
= & x^{3}-16 x
\end{aligned}
$$


Comparison with the scheme (60) shows that the coefficients of these very same polynominals appear in the full columns $0,1,2,3$, of the progressive algorithm of section VI.

\section{Solution of the Eigenvalue Problem by the Method of Minimized Iterations}

The biorthogonal property of the vector system $b_{i}, b_{i}^{*}$ leads to an explicit solution of the eigenvalue problem, in terms of the vectors $b_{i}$. Let us first assume that the matrix $A$ is of the nondefective type, and let us analyze the vectors $b_{i}$ in terms of the eigenvectors $u_{t}$. The method by which the vectors $b_{i}$ were generated, yields directly the relation

$$
b_{i}=p_{i}\left(\mu_{1}\right) u_{1}+p_{i}\left(\mu_{2}\right) u_{2}+\ldots+p_{i}\left(\mu_{m}\right) u_{m} .
$$

If this relation is dotted with $u_{k}^{*}$, we obtain, in view of the mutual orthogonality of the two sets of axes:

$$
b_{i} \cdot u_{k}^{*}=p_{i}\left(\mu_{k}\right) u_{k} \cdot u_{k}^{*} \cdot
$$

Let us now reverse the process and expand the $u_{i}$ in terms of the $b_{i}$ :

$$
u_{1}=\alpha_{i 0} b_{0}+\alpha_{i 1} b_{1}+\ldots+\alpha_{i m-1} b_{m-1} .
$$

The dotting by $b_{k}^{*}$ yields:

$$
\alpha_{i k}=\frac{u_{1} \cdot b_{k}^{*}}{b_{k} \cdot b_{k}^{*}}
$$

Let us denote the "norm" of $b_{k}$ by $\sigma_{k}$ :

$$
\sigma_{k}=b_{k} \cdot b_{k}^{*}
$$

while the norm of $u_{k}$ will be left arbitrary. Then the expansion ( 81 ) becomes:

$u_{i}=\frac{b_{0}}{\sigma_{0}}+p_{1}\left(\mu_{i}\right) \frac{b_{1}}{\sigma_{1}}+p_{2}\left(\mu_{i}\right) \frac{b_{2}}{\sigma_{2}}+\cdots+p_{m-1}\left(\mu_{i}\right) \frac{b_{m-1}}{\sigma_{m-1}}$.

This expansion contains the solution of the principal axis problem. The eigenvectors $u_{i}$ are generated in terms of the vectors $b_{1}$, which are the successive vectors of the process of minimized iterations. The expansion (84) takes the place of the previous "S-expansion" (27), which solved the eigenvector problem in terms of the customary process of iteration.

The adjoint axes are obtained in identical fashion:

$$
u_{i}^{*}=\frac{b_{0}^{*}}{\sigma_{0}}+p_{1}\left(\mu_{i}\right) \frac{b_{1}^{*}}{\sigma_{1}}+p_{2}\left(\mu_{1}\right) \frac{b_{2}^{*}}{\sigma_{2}}+\cdots+p_{m-1}\left(\mu_{i}\right) \frac{b_{s-1}^{*}}{\sigma_{m-1}}
$$

The expansion (84) remains valid even in the case of defective matrices. The only difference is that the number of principal axes becomes less than $n$ since a multiple root $\mu_{j}$, if substituted into (84) and (85) cannot contribute more than one principal axis. ${ }^{14}$ However, a defective matrix actually possesses less than $n$ pairs of principal axes, and the above expansions give the general solution of the problem.

An interesting alternative of the expansion (84) arises if we go back to the original Fredholm problem and request a solution in terms of the minimized vectors $b_{i}$, rather than the simply iterated vectors of the expansion (17). One method would be to make use of the Schmidt series (8), expressing the $u_{i}$ of that series in terms of the $b_{i}$, according to the expansion (84). However, the Schmidt series holds for nondefective matrices only, while we know that a solution must exist for any kind of matrices.

Hence, we prefer to proceed in a somewhat different fashion. Wo expand $y$ directly in terms of the vectors $b_{i}$ :

$$
y=y_{0} b_{0}+y_{1} b_{1}+\ldots+y_{m-2} b_{m-1} .
$$

We substitute this expansion into the equation (1), replacing $b_{k}^{\prime}$ by

$$
b_{k}^{\prime}=b_{k+1}+\alpha_{k} b_{k}+\beta_{k-1} b_{k-1} .
$$

Then we compare coefficients on both sides of the equation. The result can be described as follows:

Let us reverse the sequence of the $\alpha_{1}$-coefficients and let us do the same with the $\beta_{i}$-coefficients. Hence, wo define

$$
\begin{array}{ll}
\bar{\alpha}_{0}=\alpha_{m-1} & \\
\bar{\alpha}_{1}=\alpha_{m-2} & \bar{\beta}_{0}=\beta_{m-2} \\
\vdots & : \\
\bar{\alpha}_{m-1}=\alpha_{0} & \bar{\beta}_{m-2}=\beta_{0}
\end{array}
$$

16 The reader is urged to carry through the process of minlmized iterations and evaluation of the principal axes for the defective matrix

$$
\left(\begin{array}{lll}
0 & 1 & 0 \\
0 & 0 & 1 \\
0 & 0 & 0
\end{array}\right)
$$

which has only one pair of prineipal axes; (choose the trial vector in the form $b_{0}=b_{0}^{*}=1,1,1$ ). 
We now construct the following "reversed" set of polynomials:

$$
\begin{aligned}
& \bar{p}_{0} \quad=1 \\
& \bar{p}_{1}(x)=x-\bar{\alpha}_{0} \\
& \bar{p}_{2}(x)=\left(x-\bar{\alpha}_{1}\right) \bar{p}_{1}(x)-\bar{\beta}_{0} \\
& . \\
& \cdot \\
& \bar{p}_{m}(x)=\left(x-\bar{\alpha}_{m-1}\right) \bar{p}_{m-1}(x)-\bar{\beta}_{m-2} \bar{p}_{m-2}(x) \\
& \quad=G(x)
\end{aligned}
$$

Then the solution of the Fredholm problem (1) is given by the following expansion:

$y=\frac{\mu}{G(\mu)}\left[b_{m-1}+\bar{p}_{1}(\mu) b_{m-2}(u) b_{m-2}+\ldots+\bar{p}_{m-1}(u) b_{0}\right]$,

where we have put

$$
\mu=\frac{1}{\lambda}
$$

The expansion (90) is completely general and remains valid, no matter how the vector $b_{0}$ of the right side was given, and how regular or irregular the matrix $A$ may be. The only condition to be satisfied is that the vector $b_{0}^{*}$-while otherwise chosen arbitrarily-shall be free of accidental deficiencies, i. e., $b_{0}^{*}$ shall not be orthogonal to some $u_{k}$ if $b_{0}$ is not simultaneously orthogonal to $u_{\mathrm{k}}^{*}$.

The expansion (90) leads once more to a solution of the eigenvector problem, this time obtained with the help of the "reversed" polynomials $\bar{p}_{i}(x)$ :

$$
u_{t}=b_{m-1}+\bar{p}_{1}\left(\mu_{i}\right) b_{m-2}+\ldots+\bar{p}_{m-1}\left(\mu_{i}\right) b_{0} .
$$

The expansions (92) and (84) actually coincideexcept for a factor of proportionality-due to algebraic reasons.

$\left.\begin{array}{rrr}12 & 1.10638298 & 1 \\ 80 & 5.10638298 & 1 \\ -24 & -2.89361702 & 1\end{array}\right)\left(\begin{array}{cc}1 & 0 \\ 0 & 4 \\ 0 & -19.57446808\end{array}\right.$

In order to see a numericai example for this solution of the eigenvalue problem let us return once more to the simple problem of section VI. The minimized $b_{i}$ and $b_{i}^{*}$ vectors associated with this matrix were given at the end of section VII. together with the associated polynomials $p_{t}(x)$. We now construct the reversed polynomials $\bar{p}_{i}(x)$. For this purpose we tabulate the $\alpha_{i}$ and $\beta_{i}$ :

$$
\begin{array}{ll}
13 & -141 \\
-11.89361702 & 1.158895443 \\
-1.106382981 &
\end{array}
$$

We reverse the sequence of this tabulation:

$$
\begin{array}{ll}
-1.106382981 & \\
-11.89361702 & 1.58895443 \\
13 & -141
\end{array}
$$

and construct in succession:

$$
\begin{aligned}
\bar{p}_{0}=1 & \\
\bar{p}_{1}(x) & =x+1.106382981 \\
\bar{p}_{2}(x) & =(x+11.8361702) \bar{p}_{1}(x)-1.58895443 \\
& =x^{2}+13 x+12 \\
\bar{p}_{3}(x) & =(x-13) \bar{p}_{2}(x)+141 \\
& =x^{3}-16 x
\end{aligned}
$$

The last polynomial is identical with $p_{3}(x)=G(x)$. The zегоs of this polynomial are:

$$
\mu_{1}=0, \quad \mu_{2}=4, \quad \mu_{0}=-4 ;
$$

substituting these values into $\bar{p}_{2}(\mu), \bar{p}_{1}(\mu), \bar{p}_{0}$ we obtain the matrix:

$$
\left(\begin{array}{rrr}
12 & 1.10638298 & 1 \\
80 & 5.10638298 & 1 \\
-24 & -2.89361702 & 1
\end{array}\right)
$$

The product of this matrix with the matrix of the $b_{i}$ vectors gives the three principal axes $u_{i}$ :

$$
\left.\begin{array}{c}
0 \\
7 \\
4.25531914
\end{array}\right)=\left(\begin{array}{rrr}
12 & 24 & 12 \\
80 & 40 & 40 \\
-24 & 8 & -16
\end{array}\right)=\left(\begin{array}{l}
u(0) \\
u(4) \\
u(-4)
\end{array}\right)
$$


in complete agreemont with the previous result of section VI, but now obtained by an entirely different method. If the $b$-matrix is replaced by the $b^{*}$-matrix, the adjoint axes $u^{*}(0), u^{*}(4)$. $u^{*}(-4)$ are obtained.

\section{The Lateral Vibrations of a Bar}

In order to study the power of the method in connection with a vibration problem of large dispersion, the elastic vibrations of a bar were investigated. The bar was clamped at one end and free at the other. Moreover, the cross section of the bar changes in the middle, as shown in figure 1 .

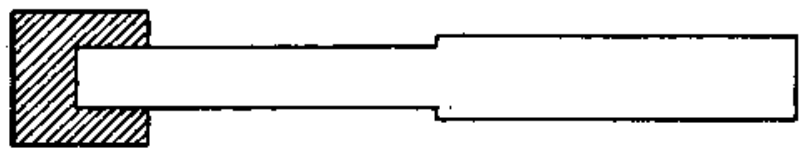

Figure 1. Elastic har, clamped at one end, free at the other; the moment of inertia makes a jump in the middle in the ratio $1: 2$.

The ehange of the cross section was such that the moment of inertia jumped from the value 1 to 2 . The differential equation that describes the vibrations of such a bar is the following fourth-order equation:

$$
\frac{d^{2}}{d x^{2}}\left[k(x) \frac{d^{2} y}{d x^{2}}\right]=\lambda y
$$

with the boundary conditions:

$$
\left.\begin{array}{rr}
y(0)=0 & y^{\prime \prime}(l)=0 \\
y^{\prime}(0)=0 & y^{\prime \prime \prime}(l)=0
\end{array}\right\}
$$

and

$$
k(x)=1\left(0 \leqq x<\frac{l}{2}\right), k(x)=2\left(\frac{l}{2}<x \leqq l\right) .
$$

The differential operator $\dot{a} / d x$ was replaced by the difference operator $\Delta / \Delta x$, with $\Delta x=1$. 'The length of the bar was chosen as $l=13$, thus leading to a 12 by 12 matrix; (since $y(0)=y(1)=0$ ).

The first step was the inversion of the matrix. This was easily accomplished, since a matrix that is composed of a narrow band around the diagonal can be inverted with little labcr. The eigenvalues $\mu_{i}$ cf the inverted matrix are the reciprocals of the original $\lambda_{i}$ :

$$
\mu_{k}=\frac{1}{\lambda_{i}}
$$

The general theory has shown that the iteration scheme applied to an arbitrary matrix automatically yields a biorthogonal set of vectors $b_{i}$ and $b_{i}^{*}$; they can be conceived as the building blocks from which the entire set of principal axes may be generated. In the present problem dissipative forces are absent, which makes the matrix $A$ symmetric and the problem self-adjoint. Hence,

$$
b_{i}=b_{i}^{*}
$$

and we get through with a single set of iterations.

Now the general procedure would demand that we go through 12 minimized iterations before the stage $b_{12}=0$ is attained. However, the study of a system with high dispersion has shown that in such a system the method of minimized iterations practically separates tho various vibrational modes, starting with the highest eigenvalue and descending systematically to the lower eigenvalues, provided that we employ a trial vector $b_{0}$ which weights the eigenvectors approximately according to the associated eigenvalues. In the present problem the trial vector $1,0,0$..., was not used directly but iterated with the matrix $A$, and then iterated again. The thus obtained vector $b_{0}^{*}$ was employed as the $b_{0}$ of the minimized iteration scheme.

The strong grading of the successive eigenvectors has the consequence that in $k$ minimized iterations essentially only the highest $k$ vibrational modes will come into evidence. This is of eminent practical value, since it allows us to dispense with the calculation of the very low eigenvalues (i. e., very high frequencies since we speak of the eigenvalues of the inverted matrix), which are often of little physical interest, and also of little mathematical interest, in view of the fact that the replacing of the $d$ operator by the $\Delta$ operator becomes in the realm of high frequencies more and more damaging.

Whether the isolation actually takes place or not can be tested with the help of the $p_{i}(x)$ polynomials, which accompany the iteration scheme. The order of these polynomials constantly inereases by 1 . The correct cigenvalues of the matrix $A$ are obtained by evaluating the zeros of the last polynomial $p_{m}(x)=0$. What actually happens, however; is that the zeros of the polynomials $p_{i}(x)$ do not change much from the beginning. If the dispersion is strong, then cach new polynomial basically adds one more root but corrects the higher roots by only small amounts. It is 
thus well possible that the series of largest roots in which we are primarily interested is practically established with sufficient accuracy after a few iterations. Then we can stop, since the later iterations will change the obtained values by negligible amounts. The same can be said about the vibrational modes associated with these roots.

This consideration suggests the following successive procedure for the approximate determination of the eigenvalues and eigenvectors (vibrational modes) of a matrix. As the minimization scheme proceeds and we constantly obtain newer and newer polynomials $p_{i}(x)$, we handle the last polynominal obtained as if it were the final polynomial $p_{m}(x)$. We evaluate the roots of this polynomial and compare them with the previous roots. Those roots that change by negligible amounts are already in their final form.

A similar procedure holds for the evaluation of the eigenvectors $u_{i}$. Here the biorthogonality of the vectors $b_{i}$ and $b_{i}^{*}$ - which is reduced to simple orthogonality in the case of a symmetric matrixis of very great help. Let us assume that the length of the vectors $b_{1}$ is normalized to 1 , by replacing $b_{i}$ by $b_{i} / \sqrt{\sigma_{i}}$. Then the expansions (84) and (85) show that the following matrix must be an orthogonal - although in the diagonal terms not normalized-matrix:

$$
\left(\begin{array}{cccc}
\frac{1}{\sqrt{\sigma_{0}}} & \frac{p_{1}\left(\mu_{1}\right)}{\sqrt{\sigma_{1}}} & \frac{p_{2}\left(\mu_{1}\right)}{\sqrt{\sigma_{2}}} & \ldots \frac{p_{m-1}\left(\mu_{1}\right)}{\sqrt{\sigma_{m-1}}} \\
\frac{1}{\sqrt{\sigma_{0}}} & \frac{p_{1}\left(\mu_{2}\right)}{\sqrt{\sigma_{1}}} & \frac{p_{2}\left(\mu_{2}\right)}{\sqrt{\sigma_{2}}} & \ldots \frac{p_{m-1}\left(\mu_{2}\right)}{\sqrt{\sigma_{m-1}}} \\
\cdot & & & \\
\cdot & & & \\
\frac{1}{\sqrt{\sigma_{0}}} & \frac{p_{1}\left(\mu_{m}\right)}{\sqrt{\sigma_{1}}} & \frac{p_{2}\left(\mu_{m}\right)}{\sqrt{\sigma_{2}}} & \ldots \frac{p_{m-1}\left(\mu_{m}\right)}{\sqrt{\sigma_{m-1}}}
\end{array}\right)
$$

The dot-product of any two rows of this matrix must come out as zero-thus providing us with a powerful check on the construction of the $p_{i}$ polynomials and the correctness of the roots $\mu_{i}$, which are the roots of the equation $p_{m}(\mu)=0 . .^{15}$ In the case of strong dispersion, the transformation matrix (98) is essentially reduced to the diagonal terms and one term to the right and to the left of the diagonal; i. e., the eigenvector $u_{k}$ is essentially a linear combination of three $b$-vectors only, viz., $b_{k-2}, b_{k-i}$. and $b_{k}$.
These general conditions are well demonstrated by the tabulation of the final results of the abovementioned bar problem. The minimized iterations were carricd through up to $m=6$. On the basis of these iterations the first six eigenvalues and the first five vibrational modes of the clampedfree bar were evaluated. The iterations were constantly watched for orthogonality. After obtaining a certain $b_{i}$, this $b_{i}$ was immediately dotted with all the previous $b_{j}$. If a certain dot-product $b_{i} \cdot b_{j}$ came out as noticeably different from zero, the correction term

$$
\epsilon_{i}=-\frac{b_{i} \cdot b_{j}}{b_{j}^{2}} b_{j}
$$

was added to $b_{i}$, thus compensating for the influence of rounding errors. By this procedure the 10 significant figure accuracy of the calculations was constantly maintained. ${ }^{10}$

The roots of the successive polynomials $p_{i}(x)$ are

\begin{tabular}{|c|c|c|}
\hline$\mu_{1}$ & $\mu_{2}$ & $\boldsymbol{\mu}_{3}$ \\
\hline $\begin{array}{r}2256.926071 \\
.943039 \\
.943939 \\
.943938 \\
.943939 \\
.943939\end{array}$ & $\begin{array}{r}48.1610705 \\
.2037755 \\
.2037825 \\
.2037825 \\
.2037825\end{array}$ & $\begin{array}{r}5.272311488 \\
.355958260 \\
356269794 \\
356269980\end{array}$ \\
\hline$\mu_{4}$ & $\mu_{6}$ & $\mu_{0}$ \\
\hline (1) & & 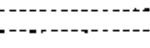 \\
\hline $\begin{array}{l}\text { 1. } 51392559 \\
.582253337 \\
.5829955952\end{array}$ & $\begin{array}{r}546827303 \\
591117817\end{array}$ & 0.2498132719 \\
\hline
\end{tabular}
tabulated as follows:

\footnotetext{
1s Due to reasons of algebre, the orthogonality of the matrix (98) bolds not only for the fleal $m$ but for any value of $m$.

is In a control experiment thst imitsted the conditions of the vibrating bar but with a more regular mstrix, the results were analytically predictsble and the cornputational results open to an exact checik. This exsmple vividaly dcrnonstrated the astonishing degree of noninterferenoe of orthogonal vectors. The spresd of the eigenvaluos was $1: 3200$. The trial vector to strongly over-emphasized the largest eigen value, containing tho lowest and the highest esgenvectors with an amplitude ratio of $1: 10^{8} ;$ (this means that if the vector of the smallest eigenvuluc were drawn with the length of 1 in., the vector of the largest efgenvalue, perpendicular to the previous one, would span the distance from Los Angeles to Chicsgo). The slightest Inclination between the two vectors would fatally injure the ehances of the smallest eigenvulue. When the entire computation scheme was fnished, the analytically required efgenvalues were computed and the comparison made. The estire set of eigenvalues, including the last, agreed with a maximum error of $z$ units in the ninth significant figure, thus demonstrating that the method is completely Iree of the cumulation of rounding errors. The suthor is indebted to Miss Fannie M. Gordon, of the National Bureau of Btandards, for the eminently eareful and skilled performance of the computing operations.
} 
The successive orthogonal transformation matrices (98) show likewise strong convergence. We tabulate here only the last computed transformation matrix (rounded off to four decimal places), which expresses the first six eigenvectors

$$
\left[\begin{array}{ccc}
1 & .0028 & 0 \\
-.0028 & 1 & .0316 \\
0 & -.0316 & 1 \\
0 & .0044 & -.1520 \\
0 & -.0010 & .0335 \\
0 & .0002 & -.0087
\end{array}\right.
$$

We notice how quickly the elements fall off to zero as soon as we are beyond one element to the right and one to the left of the main diagonal. The orthogonal reference system of the $b_{i}$ and the orthogonal reference system of the $u_{i}$ are thus in close vicinity of each other.

The obtained five vibrational modes $u_{1} \ldots, u_{5}$ ( $u_{6}$ being omitted since the lack of the neighbour on the right side of the diagonal makes the approximation unreliable) are plotted graphically in figure 2.

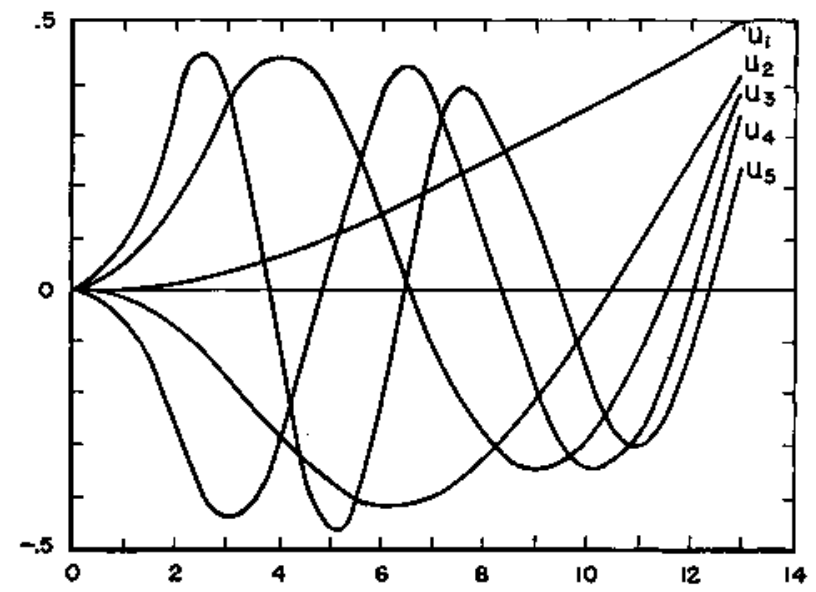

Figure 2. The first five lateral vibrational modes of the elastic bar, shown in figure 1 .

$\mu_{1}=2256.94 ; \mu_{2}=48.20 ; \mu_{3}=5.36 ; \mu_{4}=1.88 ; \mu_{5}=0.59$

\section{The Eigenvalue Problem of Linear In- tegral Operators}

The methods and results of the past sections can now be applied to the realm of continuous operators. The kernel of an integral equation can be conceived as a matrix of infinite order, which may be approximated to any degree of accuracy by a matrix of high but finite order. One method of treating an integral equation is to replace it by an ordinary matrix equation of $u_{1}, \ldots, u_{6}$ in terms of the first six normalized $b_{i} / \sqrt{\sigma_{i}}$ vectors, making use of the roots of $p_{6}(u)=0$. The diagonal elements are normalized to 1 :

$$
\left.\begin{array}{ccc}
0 & 0 & 0 \\
.0004 & 0 & 0 \\
.1497 & .0081 & 0 \\
1 & .2693 & .0249 \\
.2793 & 1 & .4033 \\
.0779 & -.3816 & 1
\end{array}\right)
$$

sufficiently high order. This procedure is, from the numerical standpoint, frequently the most satisfactory one. However, we can design methods for the solution of integral equations that obtain the solution by purely analytical tools, on the basis of an infinite convergent expansion, such as the Schmidt series, for example. The method we are going to discuss belongs to the latter type. We will find an expansion that is based on the same kind of iterative integrations as the Liouville-Neumann series, but avoiding the convergence difficulties of that expansion. The expansion we are going to develop converges under all circumstances and gives the solution of any Fredholm type of integral equation, no matter how defective the kernel of that integral equation may be. ${ }^{17}$

Let us first go back to our earlier method of solving the Fredholm problem. The solution was obtained in form of the S-expansion (17). The difficulty with this solution is that it is based on the linear identity that can be established between the iterated vectors $b_{i}$. That identity is generally of the order $n$; if $n$ grows to infinity, we have to obtain an identity of infinite order before our solution can be constructed. That, however, cannot be done without the proper adjustments.

The later attempt, based on the method of minimized iterations, employs more adequate principles. We have seen that to any matrix, $A$, a biorthogonal set of vectors $b_{i}$ and $b_{i}^{*}$ can be constructed by successive minimizations. The set is uniquely determined as soon as the first trial vectors $b_{0}$ and $b_{0}^{*}$ are given. In the case * of the inhomogeneous equation (1) the right side $b$ may be chosen as the trial vector $b_{0}$, while $b_{0}^{*}$ is still arbitrary.

17 The Volterrs type of integral equations, which have no eigonvalues and eigonsolutions, are thus included in our general considerations. 
The construction of these two sets of vectors is quite independent of the order $n$ of the matrix. If the matrix becomes an integral operator, the $b_{1}$ and $b_{i}^{*}$ vectors are transformed into a biorthogonal set of functions:

$$
\begin{array}{lll}
\phi_{0}(x), & \phi_{1}(x), & \phi_{2}(x), \ldots \\
\phi_{0}^{*}(x), & \phi_{1}^{*}(x), & \phi_{2}^{*}(x), \ldots
\end{array}
$$

which are generally present in infinite number. The process of minimized iterations assigns to $a n y$ integral operator such a set, after $\phi_{0}(x)$ and $\phi_{0}^{*}(x)$ have been chosen.

Another important feature of the process of minimized iterations was the appearance of a successive set of polynomials $p_{i}(\mu)$, tied together by the recurrence relation

$$
p_{i+1}(\mu)=\left(\mu-\alpha_{1}\right) p_{i}(\mu)-\beta_{i-1} p_{i-1}(\mu) .
$$

This is again entirely independent of the order $n$ of the matrix $A$ and remains true even if the matrix $A$ is replaced by a Fredholm kernel $K(x, \xi)$.

We can now proceed as follows. We stop at an arbitrary $p_{m}(x)$ and form the reversed set of polynomials $\vec{p}_{s}(\mu)$, defined by the process (88). Then we construct the expansion:

$$
\begin{aligned}
y_{m}(x) & =\frac{\mu}{p_{m}(\mu)}\left[\phi_{m-1}(x)+\bar{p}_{1}(\mu) \phi_{m-2}(x)+\ldots\right. \\
& \left.+\bar{p}_{m-1}(\mu) \phi_{0}(x)\right] .
\end{aligned}
$$

This gives a successive approximation process that converges well to the solution of the Fredholm integral equation

$$
y(x)-\lambda K y(x)=\phi_{0}(x) .
$$

In other words:

$$
y(x)=\lim _{m \rightarrow \infty} y_{m}(x)
$$

By the same token we can obtain all the eigenvalues and eigensolutions of the kernel $K(x, \xi)$, if such solutions exist. For this purpose we obtain the roots $\mu_{i}$ of the polynomial $p_{m}(\mu)$ by solving the algebraic equation

$$
p_{m}(\mu)=0 \text {. }
$$

The exact eigenvalues $\mu_{i}$ of the integral operator $K(x, \xi)$ are obtained by the limit process:

$$
\lim _{m \rightarrow \infty} p_{m}\left(\mu_{i}\right)=0 \text {, }
$$

where the largest root is called $\mu_{1}$, and the subsequent roots are arranged according to their absolute values. The corresponding eigenfunctions are given by the following infinite expansion:

$$
\begin{aligned}
\mu_{i}(x) & =\lim _{m \rightarrow \infty}\left[\frac{\phi_{0}(x)}{\sigma_{0}}+p_{1}\left(\mu_{i}\right) \frac{\phi_{1}(x)}{\sigma_{1}}+\cdots\right. \\
& \left.+p_{m-1}\left(\mu_{i}\right) \frac{\phi_{m-1}(x)}{\sigma_{m-1}}\right]
\end{aligned}
$$

where $\mu_{i}$ is the $i^{\text {th }}$ root of the polynomial $p_{m}(\mu) .^{18}$

As a trial function $\phi_{0}(x)$ we may choose for example

$$
\phi_{0}=\text { const. }=1 \text {. }
$$

However, the convergence is greatly speeded up if we first apply the operator $K$ to this function, and possibly iterate oven once more. In other words, we should choose $\phi_{0}=K \cdot 1$, or even $\phi_{0}=K^{2} \cdot 1$ as the basic trial function of the expansion (107).

We consider two particularly interesting examples that are well able to illustrate the nature of the successive approximation process here discussed.

(A) The vibrating membrane. In the problem of the vibrating membrane we encounter the following self-adjoint differential operator:

$$
-\frac{d}{d x} \cdot\left(x y^{\prime}\right)
$$

This leads to the eigenvalue problem

$$
-\frac{d}{d x}\left(x y^{\prime}\right)=\lambda y(0 \leqq x \leqq 1),
$$

with the boundary condition

$$
y(1)=0 \text {. }
$$

The solution of the differential equation (110) is

$$
y=J_{0}(2 \sqrt{\lambda x})
$$

wherc $J_{0}(x)$ is the Bessel-function of the order zero. The boundary condition (111) requires that $\lambda$ shall be chosen as follows:

$$
\lambda_{i}=\frac{\xi_{i}^{2}}{4}
$$

where $\xi_{i}$ are the zeros of $J_{0}(x)$.

\footnotetext{
19 This expansion is not of the nature of the Neumann series, because the coefficients of the expansion are not rigld but constantly changing with the
} number $m$ of spproximating terms. 
Now the Green's function of the differential equation (110) changes the differential operator (109) to the inverse operator, which is an integral operator of the nature of a symmetric Fredholm kerncl function $K(x, \xi)$. Our problem is to obtain the eigenvalues and eigenfunctions of this kernel.

If we start out with the function $\phi_{0}(x)=1$, the operation $K \phi_{0}$ gives

$$
1-x,
$$

and repeating the operation we obtain

$$
\frac{x^{2}}{4}-x+\frac{3}{4}
$$

and so on. The successive iterations will be polynomials in $x$. Now the minimized iterations are merely some linear combinations of the ordinary iterations. Hence the orthogonal sequence $\phi_{t}(x)$ will become a sequence of polynomials of constantly increasing order, starting with the constant $\phi_{0}=1$. This singles out the $\phi_{k}(x)$ as the Legendre polynomials $P_{k}(x)$, but normalized to the range 0 to 1 , instead of the customary range -1 to +1 . The renormalization of the range transforms the polynomials $P_{k}(x)$ into Jacobi polynomials $G_{k}(p, q ; x)$, with $p=g=1$ [8], which again are special cases of the Gaussian hypergeometric series $F(\alpha, \beta, \gamma ; x)$, in the sense of $F(k+1$, $-k, 1 ; x)$; hence, we get:

$$
\left.\begin{array}{l}
\phi_{0} \quad=1 \\
\phi_{1}(x)=1-2 x \\
\phi_{2}(x)=1-6 x+6 x^{2} \\
\phi_{0}(x)=1-12 x+30 x^{2}-20 x^{3} \\
\ldots \ldots \ldots \ldots
\end{array}\right\}
$$

The associated polynomials $p_{i}(x)$ can be obtained on the basis of the relation:

$$
K \phi_{m}=\phi_{n+1}+\alpha_{n} \phi_{n}+\beta_{n-1} \phi_{n-1} .
$$

This gives:

$$
\left.\begin{array}{l}
p_{u} \quad=1 \\
p_{1}(x)=2 x-1 \\
p_{2}(x)=24 x^{2}-18 x+1 \\
p_{3}(x)=720 x^{3}-600 x^{2}+72 x-1
\end{array}\right\}
$$

In order to obtain the general recurrence relation for these polynomials it is preferable to follow the example of the algorithm (60) and introduce the "half-columns" in addition to the full columns. Hence, we define a second set of polynomials $q_{k}(x)$ and set up the following recurrence relations:

$$
\left.\begin{array}{l}
p_{n}(x)=n x q_{n-1}(x)-p_{n-1}(x) \\
q_{n}(x)=2(2 n+1) p_{n}(x)-q_{n-1}(x)
\end{array}\right\}
$$

We thus obtain, starting with $p_{0}=1$ and $q_{0}=2$, and using successive recursions:

$$
\begin{array}{rlrl}
p_{0} & =1 & q_{0} & =2 \\
p_{1}(x) & =2 x-1 & q_{1}(x) & =12 x-8 \\
p_{2}(x) & =24 x^{2}-18 x+1 & q_{2}(x) & =240 x^{2}-192 x+18 \\
p_{3}(x) & =720 x^{3}- & q_{3}(x) & =10080 x^{3}-8640 x^{2}+ \\
600 x^{2}+72 x-1 & & 1200 x-32
\end{array}
$$

The zeros of the $p_{m}(x)$ polynomials converge to the eigenvalues of our problem, but the convergence is somewhat slow since the original function $\phi_{0}=1$ does not satisfy the boundary conditions and thus does not suppress sufficiently the eigenfunctions of high order. The zeros of the $q_{1}$ poly nomiàls give quicker convergence. They are tabulated as follows, going up to $q_{5}(x)$ :

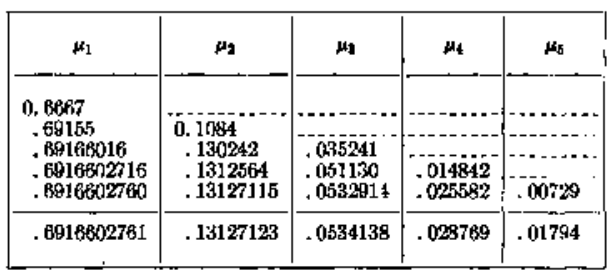

The last row contains the correct values of the eigenvalues, computed on the basis of (113):

$$
\mu_{i}=\frac{4}{\xi_{i}^{2}}
$$

We notice the excellent convergence of the scheme.

The question of the eigenfunctions of our problem will not be discussed here.

(B) The vibrating string. Even modes. Another interesting example is provided by the vibrating string. The differential operator here is 


$$
-\frac{d^{2}}{d x^{2}}
$$

with the boundary conditions

$$
y( \pm 1)=0 \text {. }
$$

The solution of the differential equation

$$
-\frac{d^{2}}{d x^{2}} y=\lambda y
$$

under the given boundary conditions is:

$$
\begin{array}{ll}
y_{t}=\cos (2 i+1) \frac{\pi}{2} x & \text { (even modes). } \\
y_{3}=\sin j \pi x & \text { (odd modes). }
\end{array}
$$

This gives the eigenvalues:

$$
\lambda_{2}=\left(\frac{2 i+1}{2} \pi\right)^{2} \quad \text { (even modes) }
$$

and

$$
\lambda_{y}=(j \pi)^{2} \quad \text { (odd modes) }
$$

If we start with the trial function $\phi_{0}=1$, we will get all the even vibrational modes of the string, while $\phi_{0}=x$ will give all the odd vibrational modes. We first choose the previous alternative.

Successive iterations give

$$
\left.\begin{array}{rl}
K \cdot 1 & =\frac{x^{2}}{2}-\frac{1}{2} \\
K^{2} \cdot 1 & =\frac{x^{4}}{24}-\frac{x^{2}}{4}+\frac{5}{24}
\end{array}\right\}
$$

and we notice that the minimized iterations will now become a sequence of even polynomials. The transformation $x^{2}=\xi$ shows that these polynomials are again Jacobi polynomials $G_{k}\left(p, q ; x^{2}\right)$, but now $p=q=\frac{1}{2}$, and we obtain the hypergeometric functions $F\left(k+\frac{1}{2},-k, \frac{1}{2} ; x^{2}\right)$ :

$$
\begin{aligned}
& \phi_{0}=1 \\
& \phi_{1}(x)=1-3 x^{2} \\
& \phi_{2}(x)=3-30 x^{2}+35 x^{4} \\
& \left.\begin{array}{l}
\phi_{3}(x)=5-105 x^{2}+315 x^{4}-231 x^{6} \\
. \quad . \quad . \quad . \quad . \quad . \quad . \quad . \quad . .
\end{array}\right\}
\end{aligned}
$$

Once more we can establish the associated polynomials $p_{i}(x)$, and the recurrence relation by which they can be generated. In the present case the recurrence relations come out as follows:

$$
\left.\begin{array}{l}
p_{n}(x)=(4 n-1) x q_{n-1}(x)-p_{n-1}(x) \\
q_{n}(x)=(4 n+1) p_{n}(x)-q_{n-1}(x)
\end{array}\right\}
$$

\begin{tabular}{|c|c|c|c|c|}
\hline$\mu_{1}$ & $\mu_{2}$ & $\mu_{3}$ & $\boldsymbol{\mu}_{\mathbf{s}}$ & $\mu_{5}$ \\
\hline \multirow{4}{*}{$\begin{array}{l}0.40000 \\
.405059 \\
.405284733 \\
.4052847346 \\
.4052847346\end{array}$} & \multirow{4}{*}{$\begin{array}{l}0.02351 \\
.044856 \\
04503010 \\
.0450316322\end{array}$} & & & \\
\hline & & & & \\
\hline & & \multirow{2}{*}{$\begin{array}{r}0.011397 \\
.015722 \\
.016192\end{array}$} & \multirow{2}{*}{$\begin{array}{r}0.00455 \\
.00752\end{array}$} & \\
\hline & & & & 0.00216 \\
\hline .4052847346 & .0450316371 & .016211 & .00827 & .00500 \\
\hline
\end{tabular}

starting with $p_{0}=1, q_{0}=1$. This yields

$$
p_{0}=1
$$

$p_{1}(x)=3 x-1$

$p_{2}(x)=105 x^{2}-45 x+1$

$p_{3}(x)=10395 x^{3}-4725 x^{2}+210 x-1$

$\begin{aligned} q_{0} & =1 \\ q_{1}(x) & =15 x-6 \\ q_{2}(x) & =945 x^{2}-420 x+15\end{aligned}$

$q_{3}(x)=135135 x^{3}-62370 x^{2}+3150 x-28$

...

The successive zeros of the $q_{1}(x)$ polynomials, up to $q_{5}(x)$, are tabulated as follows:

The last row contains the correct eigenvalues, calculated from the formula:

$$
\mu_{1}=\left(\frac{2}{2 i+1} \frac{1}{\pi}\right)^{2}
$$

The convergence is again very conspicuous.

(C) The vibrating string. Odd modes. In the case of the odd modes of the vibrating string, the orthogonal functions of the minimized iterations are again related to the Jacobi polynomials $G_{k}(p, q ; x)$, but now with $p=q=3 / 2$. Expressed in terms of the hypergeometric series we now get the polynomials of odd orders

$$
x F\left(k+\frac{3}{2},-k, \frac{3}{2}: x\right)
$$




$$
\left.\begin{array}{c}
\phi_{0}=x \\
\phi_{1}(x)=3 x-5 x^{3} \\
\phi_{2}(x)=15 x-70 x^{3}+63 x^{3} \\
\phi_{3}(x)=35 x-315 x^{3}+693 x^{5}-429 x^{7} \\
\cdots
\end{array}\right\}
$$

The associated $p_{i}(x)$ polynomials are generated by the following recurrence relations:

$$
\left.\begin{array}{l}
p_{n}(x)=(4 n+1) x q_{n-1}(x)-p_{n-1}(x) \\
q_{n}(x)=(4 n+3) p_{n}(x)-q_{n-1}(x)
\end{array}\right\}
$$

starting with $p_{0}=1, q_{0}=3$. We thus get

\begin{tabular}{|c|c|c|c|c|}
\hline$\mu_{1}$ & $\mu_{3}$ & $\mu_{3}$ & $\boldsymbol{\mu}_{4}$ & $\mu_{5}$ \\
\hline \multirow{3}{*}{$\begin{array}{l}0.0952 \\
10126 \\
.10132106 \\
.1013211836 \\
.1013211836\end{array}$} & & 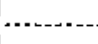 & & \\
\hline & $\begin{array}{r}02500 \\
.0100\end{array}$ & 0.00701 & & \\
\hline & $\begin{array}{l}.025323 \\
.02533024\end{array}$ & $\begin{array}{l}.01068 \\
.011215\end{array}$ & $\begin{array}{r}0.00307 \\
.00550\end{array}$ & .00158 \\
\hline .1013211836 & .025330298 & .011258 & .00633 & .00405 \\
\hline
\end{tabular}

$$
\left.\begin{array}{rl}
p_{0} & =1 \\
p_{1}(x) & =15 x-1 \\
p_{2}(x) & =945 x^{2}-105 x+1 \\
p_{3}(x) & =135135 x^{3}-17325 x^{2}+378 x-1 \\
\ldots & \\
q_{0} & =3 \quad \cdots \\
q_{1}(x) & =105 x-10 \\
q_{2}(x) & =10395 x^{2}-1260 x+21 \\
q_{3}(x) & =2027025 x^{3}-270270 x^{2}+6930 x-36
\end{array}\right\}
$$

The table of the zeros of $q_{\mathfrak{r}}(x)$, up to $q_{5}(x)$, is given as follows:

The last row contains the correct eigenvalues calculated on the basis of the formula:

$$
\mu_{i}=\frac{1}{i^{2} \pi^{2}}
$$

\section{The Eigenvalue Problem of Linear Dif- ferential Operators}

Let $D y(x)$ be a given linear differential operator, with given homogeneous boundary conditions of sufficient number to establish an eigenvalue problem. The problem of finding the eigenvalues and eigenfunctions of this operator is equivalent to the problem of the previous section in which the eigenvalue problem of linear integral operators was investigated. Let us assume that we know the Green's function $K(x, \xi)$ of the differential equation

$$
D y=\rho \text {. }
$$

Then $K$ is the reciprocal operator of $D$ which possesses the same eigenfunctions (principal axes) as the operator $D$, whereas the eigenvalues of $K$ are the reciprocals of the eigenvalues of $D$.

Hence, in principle the eigenvalue problem of differential operators needs no special investigation. Actually, however, the situation in most cases is far less simple. The assumption that we are in the possession of the Groon's function assoeiated with the differential equation (137) is often of purely theoretical significance. Even very simple differential operators have Green's functions, which are outside the limits of our analytical possibilities. Moreover, even if we do possess the integral operator $K$ in closed form, it is still possible that the successive integrations needed for the construction of the successive orthogonal functions $\phi_{1}(x), \phi_{2}(x), \ldots$. go beyond our analytical facilities.

In view of this situation we ask the question whether we could not relax some of the practically too stringent demands of the general theory. We may lose somewhat in accuracy, but we may gain tremendously in analytical operations if we can replace some of the demands of the general theory by more simplified demands. The present section will show how that may actually be accomplished.

Leaving aside the method of minimized iterations, which was merely an additional tool in our general program, the basic principle of our entire investigation, if shaped to the realm of integral operators, may be formulated as follows:

We start out with a function $f_{0}(x)$, which may be chosen as $f_{0}(x)=1$. We then form by iterated integrations a set of new functions

$$
f_{1}(x)=K f_{0}(x), f_{2}(x)=K f_{1}(x), \ldots, f_{m}(x)=K f_{m-1}(x) .
$$

Then we try to establish an approximate linear relation between these functions, as accurately as possible. For this purpose we made use of the method of least squares.

We notice that the general principle involves 
two processes: (a) the construction of the iterated set (138); (b) the establishment of a. close linear relation between them. It is the first process where the knowledge of the integral operator $K=D^{-1}$ is demanded. But let us observe that the relation between the successive $f_{\text {-functions }}$ can be stated in reverse order. We then get:

$f_{m}(x), f_{m-1}(x)=D f_{m}(x), \ldots, f_{0}(x)=D f_{1}(x)$.

If we start with the function $f_{m}(x)$, then the successive functions of lower order can be formed with the help of the given $D$ operator and we can completely dispense with the use of the Green's function.

Now the freedom of choosing $f_{0}(x)$ makes also $f_{m}(x)$ to some extent a free function. Yet, the successive functions $f_{t}(x)$ do not have the same degree of freedom. Although $f_{0}(x)$ need not satisfy the given boundary conditions, $f_{1}(x)$ of necessity satisfies these conditions, whereas $f_{2}(x)$ satisfies them more strongly, since not only $f_{2}(x)$ but even $D f_{2}(x)$ satisfies the given boundary conditions. Generally, we can say that an arbitrary $f_{n}(x)$ need not satisfy any definite differential or integral equation, but it is very restricted in the matter of boundary conditions: it has to satisfy the boundary conditions "to the $n^{\text {th }}$ order." This means that not only $f_{n}(x)$ itself, but the whole series of functions:

$$
f_{n}(x), D f_{n}(x), D^{2} f_{n}(x), \ldots D^{n-1} f_{n}(x),
$$

have to satisfy the given boundary conditions.

To construct a function $f_{n}(x)$ with this property is not too difficult. We expand $f_{n}(x)$ into a linear set of powers, or periodic functions, or any other kind of functions we may find adequate to the given problem. The coefficients of this expansion will be determined by the boundary conditions that are satisfied by $f_{n}(x)$ and the iterated functions (140). This leads to the solution of linear equations. In fact, this process can be systematized to a regular recurrence scheme that avoids the accumulation of simultaneous linear equations, replacing them by a set of separated equations, each one involving but one unknown.

We have thus constructed our set (138), although in reverse order. We did not use any integrations, only the repeated application of the given differential operator $D$. The first phase of our problem is accomplished.
We now turn to the second phase of our program, viz., the establishment of an approximate linear relation between the iterated functions $f_{i}(x)$. The method of least squares is once more at our disposal. However, here again we might encounter the difficulty that the definite integrals demanded for the evaluation of the $\alpha_{i}$ and $\beta_{i}$ are practically beyond our means. Once more we can simplify our task. The situation is similar to that of cvaluating the coefficients of a Fourier series. The "best" coefficients, obtained by the method of least squares, demand the evaluation of a set of definite integrals. Yet we can get a practically equally close approximation of a function $f(x)$ by a finite trigonometric series $\overline{f(x)}$, if we use the method of "trigonometric interpolation." Instead of minimizing the mean square of the error $f(x)-\overline{f(x)}$, we make $\overline{f(x)}$ equal to $f(x)$ at a suffieient number of equidistant points. This leads not to integrations but to simple summations.

The present situation is quite anglogous. To establish a linear relation between the $f_{i}(x)$ means that the last function $f_{m}(x)$ is to be approximated by a linear combination of the previous functions. Instead of using the method of least squares for this approximation we can use the much simpler method of interpolation, by establishing a linear relation between the successive $f_{i}(x)$ in as many equidistant points as we have coefficients at our disposal. For the sake of better convergence, it is preferable to omit $f_{0}(x)$ - which does not satisfy the boundary conditions and thus contains the high vibrational modes too pronouncedly-and establish the linear relation only from $f_{1}(x)$ on. For example, if we constructed a trial function $f_{3}(x)$ which, together with the iterated $f_{2}(x)=D f_{3}(x)$ and doubly iterated $f_{1}(x)=D^{2} f(x)$ satisfies the given boundary conditions, then we can choose two points of the region, e.g., the two endpoints, where a linear relation of the form

$$
f_{3}(x)+\alpha f_{2}(x)+\beta f_{1}(x)=0,
$$

shall hold. This gives the characteristic polynomial $G(x)$ in the form

$$
G(x)=x^{2}+\alpha x+\beta .
$$

The two roots of this polynomial give us an approximate evaluation of the two highest $\mu_{t}$, (or the two lowest $\lambda_{i}$, i,e., $\mu_{1}=1 / \lambda_{1}$, and $\mu_{i}=1 / \lambda_{2}$, 
wheress the corresponding eigensolutions are obtained by synthetic division:

$$
\left.\begin{array}{c}
\frac{G(x)}{x-\mu_{1}}=g_{1}^{\prime} x+g_{2}^{\prime} \\
\frac{G(x)}{x-\mu_{2}}=g_{1}^{\prime \prime} x+g_{2}^{\prime \prime}
\end{array}\right\}
$$

which gives

$$
\left.\begin{array}{l}
u_{1}(x)=g_{1}^{\prime} f_{2}(x)+g_{1}^{\prime} f_{1}(x) \\
u_{2}(x)=g_{1}^{\prime \prime} f_{2}(x)+g_{2}^{\prime \prime} f_{1}(x)
\end{array}\right\}
$$

(The last root and its eigenfunction are always considerably in error, and gives only rough indications.)

The remarkable feature of this method is that it completely aroids any integrations, requiring only the solution of a relatively small number of linear equations.

The following application of the method demonstrates its practical usefulness. The method was applied to obtain the first three eigenvalues of the lateral vibrations of a uniform bar, clamped at both ends. The given differential operator is here:

$$
D y=\frac{d^{4} y}{d x^{4}}
$$

with the boundary conditions

$$
y( \pm 1)=0 \quad y^{\prime}( \pm 1)=0 .
$$

Only the even modes wеге considered, expanding $y(x)$ into even powers of $x$. The approximations were carried out to first, second, and third order.

\begin{tabular}{|c|c|c|}
\hline الر & $\mu 2$ & ks \\
\hline $\begin{array}{l}0.0323413 \\
.0319686 \\
.031903958\end{array}$ & $\begin{array}{l}0.0017932 \\
.001037 b\end{array}$ & 0.0000788 \\
\hline 0. 031963996 & 0.0010946 & 0.0001795 \\
\hline
\end{tabular}
The obtained eigenvalues are tabulated in the following table, the last row containing the correct eigenvalues given by Rayleigh [9]

We notice that the general convergence behavior of this method is exactly the same as that of the analytically more advanced, but practically much more cumbersome, method of minimized iterations.

\section{Differential Equations of Second Order; Milne's Method}

If a linear differential equation of second order with two-end boundary conditions is changed into a difference equation and then handled as a matrix problem, singularly favorable conditions exist for the solution of the eigenvalue problem. The matrix of the corresponding difference equation contains only diagonal terms plus one term to the right and one to the left. If we now start to iterate with the trial vector

$$
b_{0}=1,0,0, \ldots, 0,
$$

we observe that the successive iterations grow by one element only, as indicated in the following scheme where the dots stand for the nonvanishing components:

$$
\left.\begin{array}{lllll}
b_{0}=. & & & & \\
b_{1}=. & \cdot & & & \\
b_{2}=. & \cdot & \cdot & & \\
\cdot & & & & \\
\cdot & & & & \\
b_{n-1}=. & \cdot & \cdot & \cdot & \cdot \\
b_{n}=. & \cdot & \cdot & \cdot & \cdot
\end{array}\right\}
$$

Under these conditions the establishment of the linear identity between the iterated vectors is greatly simplified since it is available by a successive recurrence scheme. The coefficients of the equation

$$
b_{n}+g_{1} b_{n-1}+g_{2} b_{n-2}+\ldots+g_{n} b_{0}=0,
$$

are directly at our disposal, since the last column of the last two vectors gives $g_{1}$, then the previous column gives $g_{2}, \ldots$, until finally the first column gives $g_{n}$. The construction of the basic polynomial $G(x)$ is thus accomplished and the cigenvalues $\lambda_{i}{ }^{10}$ directly available by finding the roots of the equation $G(\lambda)=0$.

Professor W. E. Milne of the Oregon State College and the Institute for Numerical Analysis, applied the general theory to this problem, but with the following modification. Instead of iterat-

10 We call the eigenvalues here $\lambda_{4}$, since the operator $D$ is not inverted into an integra operstor $K$. 
ing with the given matrix $A$, Milne considers the regular vibration problem

$$
\frac{\partial^{2} u}{\partial t^{2}}+D u=0
$$

where the operator $D$ has the following significance: ${ }^{20}$

$$
D u=-\left[\frac{d^{2}}{d x^{2}}+p(x) \frac{d}{d x}+g(x)\right] u(x, t) .
$$

The differential equation (148) is now converted into a difference equation, with $\Delta x=\Delta t=h$. Then the value of $u(i h, j h)$ are determined by successive recursions, starting from the initial conditions

and

$$
u(i h, 0)=1,0,0,0, \ldots, 0
$$

$$
u(i h, h)=u(i h,-h) .
$$

The linear identity between the $n+1$ vectors

$$
u(i h, 0), u(i h, h), \ldots, u(i h, n h)
$$

leads to a trigonometric equation for the characteristic frequencies $\nu_{i}$, of the following form

$$
\cos n_{\gamma_{t}} h+A_{n-1} \cos (n-1) v_{1} h+\ldots+A_{0}=0 .
$$

We then put

$$
\lambda_{i}=v_{i}^{2}
$$

On the other hand, the regular iteration method gives the eigenvalues $\bar{\lambda}_{i}$ of the operator $\Delta u$, defined in harmony with the operator $D u$ but with the modification that the operation $d / d x$ is replaced by the operation $\Delta / \Delta x$. The $\bar{\lambda}_{i}$ are in the following relation to the $\nu_{i}$ of eq 153 and 154 :

$$
\bar{\lambda}_{i}=\bar{\nu}_{i}^{2}=\left(\frac{\sin \frac{h}{2} \nu_{i}}{\frac{h}{2}}\right)^{2}
$$

It is of interest to see that the values (154) of Milne are much closer to the true eigenvalues than the values obtained by iterations. The values of Milne remain good even for high froquencies, whereas the iteration method gives gradually worse results; this is to be expected since the error committed by changing the differential equation

\footnotetext{
q0 See J. Resesreh NBE 45, 2451950 RP2132. The varisble "s" of Milnc is changed to $x$ and bis $\lambda^{2}$ to $\lambda$, to avoid conflicts with the notatlons of the present paper.
}

to a difference equation must come into evidence with ever increasing force, as we proceed to the vibrational modes of higher order.

The following table illustrates the situation. It contains the results of one of Milne's examples; ("Example 1"). Here

$$
D=-\left(\frac{d^{2}}{d x^{2}}+2 \frac{d}{d x}\right)
$$

\begin{tabular}{|c|c|c|c|}
\hline$k$ & $\sqrt{\lambda_{t}}$ & & $\sqrt{\bar{\lambda}_{k}}$ \\
\hline $\begin{array}{l}1 \\
2 \\
3 \\
4 \\
5 \\
6 \\
6\end{array}$ & $\begin{array}{r}3.2969 \\
6.3623 \\
9.4777 \\
12.60 f 1 \\
15.7398 \\
18.8761 \\
22.0139\end{array}$ & $\begin{array}{r}3.2898 \\
6.3457 \\
9.4507 \\
12.5664 \\
15.8820 \\
18.7870 \\
21.8430\end{array}$ & $\begin{array}{r}\text { 3. } 28687 \\
\text { 6. } 1806 \\
\text { R. } 9107 \\
11.3138 \\
13.3591 \\
14.75881 \\
15.6620\end{array}$ \\
\hline
\end{tabular}

with the boundary conditions:

$$
u(0)=u(1)=0 \text {. }
$$

Moreover, $h$ was chosen as $\frac{1}{8}$ and $n=7$. The column $\sqrt{\lambda_{k}}$ gives the correct frequencies, the column $\sqrt{\lambda_{k}^{*}}$ gives the frequencies obtained by Milne's method, while the column $\sqrt{\bar{\lambda}_{k}}$ gives the frequencies obtained by the iteration method.

Actually, it is purely a matter of computational preference whether we follow the one or the other scheme since there is a rigid relation between the two schemes. The frequencies $\nu_{i}$ obtained by Milne's method are in the following relation to the frequencies $\bar{\nu}_{i}$ obtained by the matrix iteration method:

$$
\nu_{i} \frac{\sin \frac{h}{2} \nu_{i}}{\frac{h}{2} \nu_{i}}=\vec{\nu}_{i}
$$

Hence, the results obtained by the one scheme can be translated into the results of the other scheme, and vice versa.

This raises the question, why it is so beneficial to transform the frequencies $\bar{y}_{i}$ of the $\Delta u$ operator to the frequencies $\nu_{i}$ by the condition

$$
\sin \frac{h}{2} \nu_{i}=\frac{h}{2} \bar{\nu}_{i}
$$

The answer is contained in the fact that the correction factor

$$
\frac{\sin \frac{h}{2} \nu_{i}}{\frac{h}{2} \nu_{i}}
$$


is exactly the factor that compensates for the transition from $d u / d x$ to $\Delta u / \Delta x$, if $u(x)$ is of the following form:

$$
u(x)=C_{i} \sin \left(\nu_{i} x+\theta_{i}\right),
$$

where the constants $C_{i}$ and $\theta_{i}$ are arbitrary.

Now it so happens that for large frequencies $v_{i}$ the first term of the operator (158) strongly overshadows the other terms. The differential equation of the eigenvalue problem for large $v_{i}$ thus becomes asymptotically:

$$
\frac{d^{2} u}{d x^{2}}+v_{i}^{2} u_{i}=0
$$

the solution of which is given by (161). This asymptotic bchavior of the solution for large frequencies makes it possible to counteract the damaging influence of the error caused by the initial transition to the difference equation. The correction is implicitly included in Milne's solution, while the results of the matrix iteration scheme can be correctod by solving eq 159 for the $\nu_{i} \cdot{ }^{21}$

\section{More-Dimensional Problems}

The present investigation was devoted to differential and integral operators that belonged to a definite finite range of the variable $x$. This variable covered a one-dimensional manifold of points. However, in many problems of physics and engineering the domain of the independent variable is more than one-dimensional. A few general remarks may be in order as to the possibility of extending the principles and methods of the present investigation to manifolds of higher dimensions.

Although the general theory of integral equations reveals that the fundamental properties of an integral equation are essentially independent of the dimensionality of the variable $x$, yet from the practical viewpoint the eigenvalue problem of higher dimensional manifolds does lead to difficulties that are not encountered in manifolds of one single dimension. The basic difference is that an essentially more-dimensional manifold of

21 This experience is valuable, since in many cigenvalue problems similar conditions hold; the elgonfunctions of large order can often be asymptotically estimated, in which case the error of the $\Delta$-procoss msy be effectively corrected. For exsmple the values $\mu_{i}$ found in section $I X$ tor the tateral vibrations of an inhomogeneous bar may bo corrected as follows:

$\mu_{i}$ uncorrected: $2256.944,48.2038,5.3563,1.5830,0.59,(0.25)$ $\mu_{i}$ corrected: $2258.824,48.4977,5.4577,1.8407,0.62,(0.28)$ eigenvalues is projected on a one-dimensional manifold, thus causing a strong overlapping of basically different vibrational modes. A good example is provided by the vibrational modes of a rectangular membrane. The eigenvalues are here given by the equation

$$
\lambda=\alpha_{1}^{2} m_{1}^{2}+\alpha_{2}^{2} m_{2}^{2}
$$

where $m_{1}$ and $m_{2}$ are two independent integers, while $\alpha_{1}^{\prime}$ and $\alpha_{2}$ are two constants determined by the length and width of the membrane.

As another illustration, consider the bewildering varicty of spectral terms that can be found within a very narrow band of frequencies, if the vibrational modes of an atom or a molecule are studied. To separate all these vibrational modes from each other poses a difficult problem that has no analogue in systems of 1 degree of freedom where the different vibrational states usually belong to well separated frequencies.

It is practically impossible to expect that one single trial function shall be sufficient for the separation of all these vibrational states. Nor does such an expectation correspond to the actual physical situation. The tremendous variety of atomic states is not excited by one single exciting function but by a rapid succession of an infinite variety of excjting functions, distributed according to some statistical probability laws. To imitate this situation mathematically means that we have to operate with a great variety of trial functions before we can hope to entangle the very dense family of vibrational states associated with a more than one-dimensional manifold.

In this connection it seems appropriate to say a word about the physical significance of the "trial function" $\phi_{0}(x)$ that we have employed for the generation of an entire system of eigenfunctions. At first sight this trial function may appear as a purely mathematical quantity that has no analogue in the physical world. The homogeneous integral equation that defines the eigenvalues, and the eigenfunctions, of a given integral operator, does not come physically into evidence since in the domain of physical reality there is always a "driving force" that provides the right side of the integral equation; it is thus the inhomogeneous and not the homogeneous equation that has direct physical significance.

If we carefully analyze the method of successive approximations by which the eigenvalues and the eigenfunctions of a given integral operator were obtained, we cannot fail to observe that we have 
basically operated with the inhomogeneous equation (103) and our trial function $\phi_{0}(x)$ serves merely as the "exciting function" or "driving force." Indeed, the solution (107) for the eigenfunctions is nothing but a special case of the general solution (102), but applied to such values of the parameter $\lambda$, which make the denominator zero. This means that we artificially generate the state of "resonance", which singles out one definite eigenvalue $\lambda_{i}$ and its associated eigenfunction $\phi_{i}(x)$.

From this point of view we can say that, while the separation of all the cigenfunctions of a multidimensional operator might be a practically insuperable task-except if the technique of "separation" is applicable, which reduces the moredimensional problem to a succession of onedimensional problems-yet it might not be too difficult to obtain the solution of a given moredimensional integral equation if the right side (i. e., physically, the "driving force") is given as a sufficiently smooth function that does not contain a too-large variety of eigenfunctions. Then the convergenee of the method may still suffice for a solution that gives the output function with a practically satisfactory accuracy. This is the situation in many antenna and wave-guide problems that are actually input-output problems, rather than strict resonance problems. In other words, what we want to get is a certain mixture of weighted eigenfunctions, which appear physically together, on account of the exciting mechanism, while the isolation of each eigenfunction for itself is not demanded. Problems of this type are much more amenable to a solution than problems that demand a strict separation of the infinite variety of eigenfunctions associated with a multi-dimensional differential or integral operator. To show the applicability of the method to problems of this nature will be the task of a future investigation.

\section{Summary}

The present investigation establishes a systematic procedure for the evaluation of the latent roots and principal axes of a matrix, without constant reductions of the order of the matrix. A systematic algorithm (called "progressive algorithm") is developed, which obtains the linear identity between the iterated vectors in successive steps by means of recursions. The accuracy of the obtained relation increases constantly, until in the end full accuracy is obtained.

This procedure is then modified to the method of "minimized iterations", in order to avoid the accumulation of rounding errors. Great accuracy is thus obtainable even in the case of matrices that exhibit a large dispersion of the eigenvalues. Moreover, the good convergence of the method in the case of large dispersion makes it possible to operate with a small number of iterations, obtaining $m$ successive eigenvalues and principal axes by only $m+1$ iterations.

These results are independent of the order of the matrix and can thus be immediately applied to the realm of differential and integral operators. This results in a well-convergent approximation method by which the solution of an integral equation of the Fredholm type is obtained by successive iterations. The same procedure obtains the eigenvalues and eigensolutions of the given integral operator, if these eigensolutions exist.

In the case of differential operators, the toostringent demands of the least square mothod may be relaxed. The approximate linear identity between the iterated functions may be established by interpolation, thus dispensing with the evaluation of definite integrals. Moreover, the iterations may be carried out with the given differential operator itself, instead of reverting to the Green's function, which is frequently not avalable in closed form. The entire procedure is then free of integrations and requires only the solution of linear equations.

The present investigation contains the results of years of research in the fields of network analysis, flutter problems, vibration of antennas, solution of systems of linear equations, encountered by the author in his consulting and research work for the Boeing Airplane Co., Seattle, Wash. The final conclusions were reached since the author's stay with the Institute for Numerical Analysis, of the National Bureau of Standards. The author expresses his heartfelt thanks to $\mathbf{C}$. $K$. Stedman, head of the Physical Research Unit of the Boeing Airplane Co. and to J. H. Curtiss, Acting Director of the Institute for Numerical Analysis, for the generous support of his scientific endeavors. 


\section{References}

[1] H. Hotelling, Psychometrika 1, 27 to 35 (1936).

[2] A. C. Aitken, Proc. Roy. Soc. Edinburgh 57, 269 to 304 (1937).

[3] W. Wayland, Quarterly of Appl. Math. 2, 277 to 306 (1945).

[4] W. U. Kincaid, Quarterly of Appl. Math. 5, 320 to 345 (1947).

[5] I. Fredholm, Acta Math, 27, 365 to 390 (1903).

[6] Whittaker-Watson, Modern analysis, 4th ed., p. 221 (Cambridge Univ. Press, 1935).
[7] Whittaker-Watson, Modern analysis, 4th ed., p. 228 (Cambridge Univ. Press, 1935); Courant-Hilbert, Methoden d. math. Physik. I, 116 (J. Springer, Berlin, 1931).

[8] Courant-Hilbert, Methoden d. math. Physik. I, 76 (J. Springer, Berlin, 1931).

[9] Lord Rayleigh, The theory of sound 1, 272 to 278 (reprinted; Dover Publications, New York, N. Y., 1945).

Los Angeles, September, 1949. 